\title{
FREE RESOLUTIONS OF SOME SCHUBERT SINGULARITIES.
}

\author{
MANOJ KUMMINI, V. LAKSHMIBAI, PRAMATHANATH SASTRY, AND C. S. SESHADRI
}

\begin{abstract}
In this paper we construct free resolutions of certain class of closed subvarieties of affine spaces (the so-called "opposite big cells" of Grassmannians). Our class covers the determinantal varieties, whose resolutions were first constructed by A. Lascoux (Adv. Math., 1978). Our approach uses the geometry of Schubert varieties. An interesting aspect of our work is its connection to the computation of the cohomology of homogeneous bundles (that are not necessarily completely reducible) on partial flag varieties.
\end{abstract}

\section{INTRODUCTION}

A classical problem in commutative algebra and algebraic geometry is to describe the syzygies of defining ideals of interesting varieties. Let $k \leq n \leq m$ be positive integers. The space $D_{k}$ of $m \times n$ matrices (over a field $\mathbb{k}$ ) of rank at most $k$ is a closed subvariety of the $m n$-dimensional affine space of all $m \times n$ matrices. When $\mathbb{k}=\mathbb{C}$, a minimal free resolution of the coordinate ring $\mathbb{k}\left[\mathscr{O}_{D_{k}}\right]$ as a module over the coordinate ring of the the $m n$-dimensional affine space (i.e. the $m n$-dimensional polynomial ring) was constructed by A. Lascoux [Las78]; see also [Wey03, Chapter 6].

In this paper, we construct free resolutions for a larger class of singularities, viz., Schubert singularities, i.e., the intersection of a singular Schubert variety and the "opposite big cell" inside a Grassmannian. The advantage of our method is that it is algebraic group-theoretic, and is likely to work for Schubert singularities in more general flag varieties. In this process, we have come up with a method to compute the cohomology of certain homogeneous vector-bundles (which are not completely reducible) on flag varieties. We will work over $\mathbb{k}=\mathbb{C}$.

Let $N=m+n$. Let $\mathrm{GL}_{N}=\mathrm{GL}_{N}(\mathbb{C})$ be the group of $N \times N$ invertible matrices. Let $B_{N}$ be the Borel subgroup of all upper-triangular matrices and $B_{N}^{-}$the opposite Borel subgroup of all lowertriangular matrices in $\mathrm{GL}_{N}$. Let $P$ be the maximal parabolic subgroup corresponding to omitting the simple root $\alpha_{n}$, i.e, the subgroup of $\mathrm{GL}_{N}$ comprising the matrices in which the $(i, j)$-th entry (i.e., in row $i$ and column $j$ ) is zero, if $n+1 \leq i \leq N$ and $1 \leq j \leq n$; in other words,

$$
P=\left\{\left[\begin{array}{cc}
A_{n \times n} & C_{n \times m} \\
0_{m \times n} & E_{m \times m}
\end{array}\right] \in \mathrm{GL}_{N}\right\} .
$$

We have a canonical identification of the Grassmannian of $n$-dimensional subspaces of $\mathbb{k}^{N}$ with $\mathrm{GL}_{N} / P$. Let $W$ and $W_{P}$ be the Weyl groups of $\mathrm{GL}_{N}$ and of $P$, respectively; note that $W=S_{N}$ (the symmetric group) and $W_{P}=S_{n} \times S_{m}$. For $w \in W / W_{P}$, let $X_{P}(w) \subseteq \mathrm{GL}_{N} / P$ be the Schubert variety corresponding to $w$ (i.e., the closure of the $B_{N}$-orbit of the coset $w P\left(\in \mathrm{GL}_{N} / P\right)$, equipped with the canonical reduced scheme structure). The $B_{N}^{-}$-orbit of the coset (id $\cdot P$ ) in $\mathrm{GL}_{N} / P$ is denoted by $O_{\mathrm{GL}}^{-} / P$, and is usually called the opposite big cell in $\mathrm{GL}_{N} / P$; it can be identified with the $m n$-dimensional affine space. (See 2.2.)

Write $W^{P}$ for the set of minimal representatives (under the Bruhat order) in $W$ for the elements of $W / W_{P}$. For $1 \leq r \leq n-1$, we consider certain subsets $\mathcal{W}_{r}$ of $W^{P}$ (Definition 3.11); there is

The first author was supported by a CMI Faculty Development Grant. The second author was supported by NSA grant H98230-11-1-0197, NSF grant 0652386. 
$w \in \mathcal{W}_{n-k}$ such that $D_{k}=X_{P}(w) \cap O_{\mathrm{GL}_{N} / P}^{-}$. Note that for any $w \in W^{P}, X_{P}(w) \cap O_{\mathrm{GL}_{N} / P}^{-}$is a closed subvariety of $O_{\mathrm{GL}_{N} / P}^{-}$. Our main result is a description of the minimal free resolution of the coordinate ring of $X_{P}(w) \cap O_{\mathrm{GL}_{N} / P}^{-}$as a module over the coordinate ring of $O_{\mathrm{GL}_{N} / P}^{-}$for every $w \in \mathcal{W}_{r}$. This latter ring is a polynomial ring. We now outline our approach.

First we recall the Kempf-Lascoux-Weyman "geometric technique" of constructing minimal free resolutions. Suppose that we have a commutative diagram of varieties

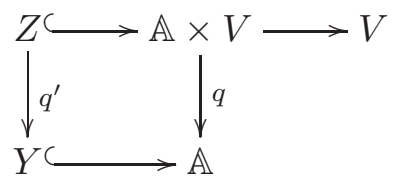

where $\mathbb{A}$ is an affine space, $Y$ a closed subvariety of $\mathbb{A}$ and $V$ a projective variety. The map $q$ is first projection, $q^{\prime}$ is proper and birational, and the inclusion $Z \hookrightarrow \mathbb{A} \times V$ is a sub-bundle (over $V$ ) of the trivial bundle $\mathbb{A} \times V$. Let $\xi$ be the dual of the quotient bundle on $V$ corresponding to $Z$. Then the derived direct image $\mathbf{R} q_{*}^{\prime} \mathscr{O}_{Z}$ is quasi-isomorphic to a minimal complex $F_{\bullet}$ with

$$
F_{i}=\oplus_{j \geq 0} \mathrm{H}^{j}\left(V, \bigwedge^{i+j} \xi\right) \otimes_{\mathbb{C}} R(-i-j) .
$$

Here $R$ is the coordinate ring of $\mathbb{A}$; it is a polynomial ring and $R(k)$ refers to twisting with respect to its natural grading. If $q^{\prime}$ is such that the natural map $\mathscr{O}_{Y} \longrightarrow \mathbf{R} q_{*}^{\prime} \mathscr{O}_{Z}$ is a quasi-isomorphism, (for example, if $q^{\prime}$ is a desingularization of $Y$ and $Y$ has rational singularities), then $F_{\bullet}$ is a minimal free resolution of $\mathbb{C}[Y]$ over the polynomial ring $R$.

The difficulty in applying this technique in any given situation is two-fold: one must find a suitable morphism $q^{\prime}: Z \longrightarrow Y$ such that the map $\mathscr{O}_{Y} \longrightarrow \mathbf{R} q_{*}^{\prime} \mathscr{O}_{Z}$ is a quasi-isomorphism and such that $Z$ is a vector-bundle over a projective variety $V$; and, one must be able to compute the necessary cohomology groups. We overcome this for opposite cells in a certain class (which includes the determinantal varieties) of Schubert varieties in a Grassmannian, in two steps.

As the first step, we need to establish the existence of a diagram as above. This is done using the geometry of Schubert varieties. We take $\mathbb{A}=O_{\mathrm{GL}_{N} / P}^{-}$and $Y=Y_{P}(w):=X_{P}(w) \cap O_{\mathrm{GL}_{N} / P^{-}}^{-}$. Let $\tilde{P}$ be a parabolic subgroup with $B_{N} \subseteq \tilde{P} \subsetneq P$. The inverse image of $O_{\mathrm{GL}_{N} / P}^{-}$under the natural $\operatorname{map} \mathrm{GL}_{N} / \tilde{P} \longrightarrow \mathrm{GL}_{N} / P$ is $O_{\mathrm{GL}_{N} / P}^{-} \times P / \tilde{P}$. Let $\tilde{w}$ be the representative of the coset $w \tilde{P}$ in $W^{\tilde{P}}$. Then $X_{\tilde{P}}(\tilde{w}) \subseteq \mathrm{GL}_{N} / \tilde{P}$ (the Schubert subvariety of $\mathrm{GL}_{N} / \tilde{P}$ associated to $\tilde{w}$ ) maps properly and birationally onto $X_{P}(w)$. We may choose $\tilde{P}$ to ensure that $X_{\tilde{P}}(\tilde{w})$ is smooth. Let $Z_{\tilde{P}}(\tilde{w})$ be the preimage of $Y_{P}(w)$ in $X_{\tilde{P}}(\tilde{w})$. We take $Z=Z_{\tilde{P}}(\tilde{w})$. Then $V$, which is the image of $Z$ under the second projection, is a smooth Schubert subvariety of $P / \tilde{P}$. The vector-bundle $\xi$ on $V$ that we obtain is the restriction of a homogeneous bundle on $P / \tilde{P}$. Thus we get:

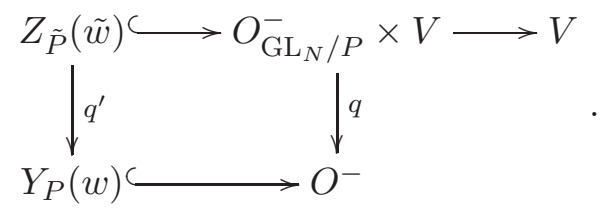

See Theorem 3.7 and Corollary 3.9. In this diagram, $q^{\prime}$ is a desingularization of $Y_{P}(w)$. Since it is known that Schubert varieties have rational singularities, we have that the map $\mathscr{O}_{Y} \longrightarrow \mathbf{R} q_{*}^{\prime} \mathscr{O}_{Z}$ is a quasi-isomorphism, so $F_{\bullet}$ is a minimal resolution.

As the second step, we need to determine the cohomology of the homogeneous bundles $\wedge^{t} \xi$ over $V$. There are two ensuing issues: computing cohomology of homogeneous vector-bundles over 
Schubert subvarieties of flag varieties is difficult and, furthermore, these bundles are not usually completely reducible, so one cannot apply the Borel-Weil-Bott theorem directly. We address the former issue by restricting our class; if $w \in \mathcal{W}_{r}$ (for some $r$ ) then $V$ will equal $P / \tilde{P}$. Regarding the latter issue, we inductively replace $\tilde{P}$ by larger parabolic subgroups (still inside $P$ ), such that at each stage, the computation reduces to that of the cohomology of completely reducible bundles on Grassmannians; using various spectral sequences, we are able to determine the cohomology groups that determine the minimal free resolution. See Proposition 5.5 for the key inductive step. In contrast, in Lascoux's construction of the resolution of determinantal ideals, one comes across only completely reducible bundles; therefore, one may use the Borel-Weil-Bott theorem to compute the cohomology of the bundles $\wedge^{t} \xi$.

Computing cohomology of homogeneous bundles, in general, is difficult, and is of independent interest; we hope that our approach would be useful in this regard. The best results, as far as we know, are due to G. Ottaviani and E. Rubei [OR06], which deal with general homogeneous bundles on Hermitian symmetric spaces. The only Hermitian symmetric spaces in Type A are the Grassmannians, so their results do not apply to our situation.

Since the opposite big cell $O_{\mathrm{GL}_{N} / P}^{-}$intersects every $B_{N}$-orbit of $\mathrm{GL}_{N} / P, Y_{P}(w)$ captures all the singularities of $X_{P}(w)$ for every $w \in W$. In this paper, we describe a construction of a minimal free resolution of $\mathbb{C}\left[Y_{P}(w)\right]$ over $\mathbb{C}\left[O_{\mathrm{GL}_{N} / P}^{-}\right]$. We hope that our methods could shed some light on the problem of construction of a locally free resolution of $\mathscr{O}_{X_{P}(w)}$ as an $\mathscr{O}_{\mathrm{GL}_{N} / P^{-}}$-module.

The paper is organized as follows. Section 2 contains notations and conventions (Section 2.1) and the necessary background material on Schubert varieties (Section 2.2) and homogeneous bundles (Section 2.3). In Section 3, we discuss properties of Schubert desingularization, including the construction of Diagram (1.2). Section 4 is devoted to a review of the Kempf-Lascoux-Weyman technique and its application to our problem. Section 5 explains how the cohomology of the homogeneous bundles on certain partial flag varieties can be computed; Section 6 gives some examples. Finally, in Section 7, we describe Lascoux's resolution in terms of our approach and describe the multiplicity and Castelnuovo-Mumford regularity of $\mathbb{C}\left[Y_{P}(w)\right]$.

\section{ACKNOWLEDGEMEnTS}

Most of this work was done during a visit of the first author to Northeastern University and the visits of the second author to Chennai Mathematical Institute; the two authors would like to thank the respective institutions for the hospitality extended to them during their visits. The authors thank V. Balaji, Reuven Hodges and A. J. Parameswaran for helpful comments. The computer algebra systems Macaulay2 [M2] and LiE [LiE] provided valuable assistance in studying examples.

\section{Preliminaries}

In this section, we collect various results about Schubert varieties, homogeneous bundles and the Kempf-Lascoux-Weyman geometric technique.

2.1. Notation and conventions. We collect the symbols used and the conventions adopted in the rest of the paper here. For details on algebraic groups and Schubert varieties, the reader may refer to [Bor91, Jan03, BL00, Ses07].

Let $m \geq n$ be positive integers and $N=m+n$. We denote by $\mathrm{GL}_{N}$ (respectively, $B_{N}, B_{N}^{-}$) the group of all (respectively, upper-triangular, lower-triangular) invertible $N \times N$ matrices over C. The Weyl group $W$ of $\mathrm{GL}_{N}$ is isomorphic to the group $S_{N}$ of permutations of $N$ symbols and is generated by the simple reflections $s_{i}, 1 \leq i \leq N-1$, which correspond to the transpositions $(i, i+1)$. For $w \in W$, its length is the smallest integer $l$ such that $w=s_{i_{1}} \cdots s_{i_{l}}$ as a product of 
simple reflections. For every $1 \leq i \leq N-1$, there is a minimal parabolic subgroup $P_{i}$ containing $s_{i}$ (thought of as an element of $\mathrm{GL}_{N}$ ) and a maximal parabolic subgroup $P_{\hat{i}}$ not containing $s_{i}$. Any parabolic subgroup can be written as $P_{\widehat{A}}:=\bigcap_{i \in A} P_{\widehat{i}}$ for some $A \subset\{1, \ldots, N-1\}$. On the other hand, for $A \subseteq\{1, \ldots, N-1\}$ write $P_{A}$ for the subgroup of $\mathrm{GL}_{N}$ generated by $P_{i}, i \in A$. Then $P_{A}$ is a parabolic subgroup and $P_{\{1, \ldots, N-1\} \backslash A}=P_{\widehat{A}}$.

The following is fixed for the rest of this paper:

(a) $P$ is the maximal parabolic subgroup $P_{\widehat{n}}$ of $\mathrm{GL}_{N}$;

(b) for $1 \leq s \leq n-1, \tilde{P}_{s}$ is the parabolic subgroup $P_{\{1, \ldots, s-1, n+1, \ldots, N-1\}}=\cap_{i=s}^{n} P_{i}$ of $\mathrm{GL}_{N}$;

(c) for $1 \leq s \leq n-1, Q_{s}$ is the parabolic subgroup $P_{\{1, \ldots, s-1\}}=\cap_{i=s}^{n-1} P_{\hat{i}}$ of $\mathrm{GL}_{n}$.

We write the elements of $W$ in one-line notation: $\left(a_{1}, \ldots, a_{N}\right)$ is the permutation $i \mapsto a_{i}$. For any $A \subseteq\{1, \ldots, N-1\}$, define $W_{P_{A}}$ to be the subgroup of $W$ generated by $\left\{s_{i}: i \in A\right\}$. By $W^{P_{A}}$ we mean the subset of $W$ consisting of the minimal representatives (under the Bruhat order) in $W$ of the elements of $W / W_{P_{A}}$. For $1 \leq i \leq N$, we represent the elements of $W^{P_{i}}$ by sequences $\left(a_{1}, \ldots, a_{i}\right)$ with $1 \leq a_{1}<\cdots<a_{i} \leq N$ since under the action of the group $W_{P_{\vec{i}}}$, every element of $W$ can be represented minimally by such a sequence.

For $w=\left(a_{1}, a_{2}, \ldots, a_{n}\right) \in W^{P}$, let $r(w)$ be the integer $r$ such that $a_{r} \leq n<a_{r+1}$.

We identify $\mathrm{GL}_{N}=\mathrm{GL}(V)$ for some $N$-dimensional vector-space $V$. Let $A:=\left\{i_{1}<i_{2}<\cdots<\right.$ $\left.i_{r}\right\} \subseteq\{1, \ldots, N-1\}$. Then $\mathrm{GL}_{N} / P_{\widehat{A}}$ is the set of all flags $0=V_{0} \subsetneq V_{1} \subsetneq V_{2} \subsetneq \cdots \subsetneq V_{r} \subsetneq V$ of subspaces $V_{j}$ of dimension $i_{j}$ inside $V$. We call $\mathrm{GL}_{N} / P_{\widehat{A}}$ a flag variety. If $A=\{1, \ldots, N-1\}$ (i.e. $\left.P_{\widehat{A}}=B_{N}\right)$, then we call the the flag variety a full flag variety; otherwise, a partial flag variety. The Grassmannian $\mathrm{Gr}_{i, N}$ of $i$-dimensional subspaces of $V$ is $\mathrm{GL}_{N} / P_{\hat{i}}$.

Let $\tilde{P}$ be any parabolic subgroup containing $B_{N}$ and $\tau \in W$. The Schubert variety $X_{\tilde{P}}(\tau)$ is the closure inside $\mathrm{GL}_{N} / \tilde{P}$ of $B_{N} \cdot e_{w}$ where $e_{w}$ is the coset $\tau \tilde{P}$, endowed with the canonical reduced scheme structure. Hereafter, when we write $X_{\tilde{P}}(\tau)$, we mean that $\tau$ is the representative in $W^{\tilde{P}}$ of its coset. The opposite big cell $O_{\mathrm{GL}_{N} / \tilde{P}}^{-}$in $\mathrm{GL}_{N} / \tilde{P}$ is the $B_{N}^{-}$-orbit of the coset (id $\left.\cdot \tilde{P}\right)$ in $\mathrm{GL}_{N} / \tilde{P}$. Let $Y_{\tilde{P}}(\tau):=X_{\tilde{P}}(\tau) \cap O_{\mathrm{GL}_{N} / \tilde{P}}^{-}$; we refer to $Y_{\tilde{P}}(\tau)$ as the opposite cell of $X_{\tilde{P}}(\tau)$.

We will write $R^{+}, R^{-}, R_{\tilde{P}}^{+}, R_{\tilde{P}}^{-}$, to denote respectively, positive and negative roots for GL $\mathrm{G}_{N}$ and for $\tilde{P}$. We denote by $\epsilon_{i}$ the character that sends the invertible diagonal matrix with $t_{1}, \ldots, t_{n}$ on the diagonal to $t_{i}$.

2.2. Précis on $\mathrm{GL}_{n}$ and Schubert varieties. Let $\tilde{P}$ be a parabolic subgroup of $\mathrm{GL}_{N}$ with $B_{N} \subseteq \tilde{P} \subseteq P$. We will use the following proposition extensively in the sequel.

Proposition 2.2.1. Write $U_{\tilde{P}}^{-}$for the negative unipotent radical of $\tilde{P}$.

(a) $O_{\mathrm{GL}_{N} / \tilde{P}}^{-}$can be naturally identified with $U_{\tilde{P}}^{-} \tilde{P} / \tilde{P}$.

(b) For

$$
z=\left[\begin{array}{cc}
A_{n \times n} & C_{n \times m} \\
D_{m \times n} & E_{m \times m}
\end{array}\right] \in \mathrm{GL}_{N}
$$

$z P \in O_{\mathrm{GL}_{N} / P}^{-}$if and only if $A$ is invertible.

(c) For $1 \leq s \leq n-1$, the inverse image of $O_{\mathrm{GL}_{N} / P}^{-}$under the natural map $\mathrm{GL}_{N} / \tilde{P}_{s} \longrightarrow \mathrm{GL}_{N} / P$ is isomorphic to $O_{\mathrm{GL}_{N} / P}^{-} \times P / \tilde{P}_{s}$ as schemes. Every element of $O_{\mathrm{GL}_{N} / P}^{-} \times P / \tilde{P}_{s}$ is of the form

$$
\left[\begin{array}{cc}
A_{n \times n} & 0_{n \times m} \\
D_{m \times n} & I_{m}
\end{array}\right] \quad \bmod \tilde{P}_{s} \in \mathrm{GL}_{N} / \tilde{P}_{s} .
$$


FREE RESOLUTIONS OF SOME SCHUBERT SINGULARITIES.

Moreover, two matrices

$$
\left[\begin{array}{cc}
A_{n \times n} & 0_{n \times m} \\
D_{m \times n} & I_{m}
\end{array}\right] \text { and }\left[\begin{array}{cc}
A_{n \times n}^{\prime} & 0_{n \times m} \\
D_{m \times n}^{\prime} & I_{m}
\end{array}\right]
$$

in $\mathrm{GL}_{N}$ represent the same element modulo $\tilde{P}_{s}$ if and only if there exists a matrix $q \in Q_{s}$ such that $A^{\prime}=A q$ and $D^{\prime}=D q$.

(d) For $1 \leq s \leq n-1, P / \tilde{P}_{s}$ is isomorphic to $\mathrm{GL}_{n} / Q_{s}$. In particular, the projection map $O_{\mathrm{GL}_{N} / P}^{-} \times P / \tilde{P} \longrightarrow P / \tilde{P}_{s}$ is given by

$$
\left[\begin{array}{cc}
A_{n \times n} & 0_{n \times m} \\
D_{m \times n} & I_{m}
\end{array}\right] \quad \bmod \tilde{P}_{s} \mapsto A \quad \bmod \tilde{Q} \in \mathrm{GL}_{n} / Q \simeq P / \tilde{P}_{s}
$$

Proof. (a): Note that $U_{\tilde{P}}^{-}$is the subgroup of $\mathrm{GL}_{N}$ generated by the (one-dimensional) root subgroups $\overline{U_{\alpha}}, \alpha \in R^{-} \backslash R_{\tilde{P}}^{-}$and that $U_{\tilde{P}}^{-} \tilde{P} / \tilde{P}=B_{N}^{-} \tilde{P} / \tilde{P}$. Hence under the canonical projection $\mathrm{GL}_{N} \longrightarrow \mathrm{GL}_{N} / P, g \mapsto g P, U_{P}^{-}$is mapped onto $O_{\mathrm{GL}_{N} / \tilde{P}}^{-}$. It is easy to check that this is an isomorphism.

(b): Suppose that $z P \in O_{\mathrm{GL}_{N} / P}^{-}$. By (a), we see that there exist matrices $A_{n \times n}^{\prime}, C_{n \times m}^{\prime}, D_{m \times n}^{\prime}$ and $E_{m \times m}^{\prime}$ such that

$$
z_{1}:=\left[\begin{array}{cc}
I_{n} & 0_{n \times m} \\
D_{m \times n}^{\prime} & I_{m}
\end{array}\right] \in U_{P}^{-}, z_{2}:=\left[\begin{array}{cc}
A_{n \times n}^{\prime} & C_{n \times m}^{\prime} \\
0_{m \times n} & E_{m \times m}^{\prime}
\end{array}\right] \in P \text { and } z=\left[\begin{array}{cc}
A_{n \times n} & C_{n \times m} \\
D_{m \times n} & E_{m \times m}
\end{array}\right]=z_{1} z_{2} .
$$

Hence $A=A^{\prime}$ is invertible. Conversely, if $A$ is invertible, then we may write $z=z_{1} z_{2}$ where

$$
z_{1}:=\left[\begin{array}{cc}
I_{n} & 0 \\
D A^{-1} & I_{m}
\end{array}\right] \in U_{P}^{-} \text {and } z_{2}:=\left[\begin{array}{cc}
A & C \\
0 & E-D A^{-1} C
\end{array}\right] \text {. }
$$

Since $z \in \mathrm{GL}_{N}, z_{2} \in P$.

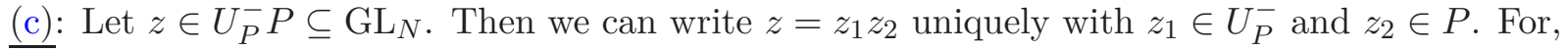
suppose that

$$
\left[\begin{array}{cc}
I_{n} & 0_{n \times m} \\
D_{m \times n} & I_{m}
\end{array}\right]\left[\begin{array}{cc}
A_{n \times n} & C_{n \times m} \\
0_{m \times n} & E_{m \times m}
\end{array}\right]=\left[\begin{array}{cc}
I_{n} & 0_{n \times m} \\
D_{m \times n}^{\prime} & I_{m}
\end{array}\right]\left[\begin{array}{cc}
A_{n \times n}^{\prime} & C_{n \times m}^{\prime} \\
0_{m \times n} & E_{m \times m}^{\prime}
\end{array}\right]
$$

then $A=A^{\prime}, C=C^{\prime}, D A=D^{\prime} A^{\prime}$ and $D C+E=D^{\prime} C^{\prime}+E^{\prime}$, which yields that $D^{\prime}=D$ (since $A=A^{\prime}$ is invertible, by (b)) and $E=E^{\prime}$. Hence $U_{P}^{-} \times_{\mathbb{C}} P=U_{P}^{-} P$. Therefore, for any parabolic subgroup $P^{\prime} \subseteq P, U_{P}^{-} \times_{\mathbb{C}} P / P^{\prime}=U_{P}^{-} P / P^{\prime}$. The asserted isomorphism now follows by taking $P^{\prime}=\tilde{P}_{s}$.

For the next statement, let

$$
\left[\begin{array}{cc}
A_{n \times n} & C_{n \times m} \\
D_{m \times n} & E_{m \times m}
\end{array}\right] \in \mathrm{GL}_{N}
$$

with $A$ invertible (which we may assume by (b)). Then we have a decomposition (in $\mathrm{GL}_{N}$ )

$$
\left[\begin{array}{cc}
A & C \\
D & E
\end{array}\right]=\left[\begin{array}{cc}
A & 0_{n \times m} \\
D & I_{m}
\end{array}\right]\left[\begin{array}{cc}
I_{n} & A^{-1} C \\
0_{m \times n} & E-D A^{-1} C
\end{array}\right] .
$$

Hence

$$
\left[\begin{array}{cc}
A & C \\
D & E
\end{array}\right] \equiv\left[\begin{array}{cc}
A & 0_{n \times m} \\
D & I_{m}
\end{array}\right] \quad \bmod \tilde{P}_{s}
$$

Finally,

$$
\left[\begin{array}{cc}
A_{n \times n} & 0_{n \times m} \\
D_{m \times n} & I_{m}
\end{array}\right] \equiv\left[\begin{array}{cc}
A_{n \times n}^{\prime} & 0_{n \times m} \\
D_{m \times n}^{\prime} & I_{m}
\end{array}\right] \quad \bmod \tilde{P}_{s}
$$


if and only if there exist matrices $q \in Q_{s}, q_{n \times m}^{\prime}$ and $\tilde{q}_{n \times n} \in \mathrm{GL}_{m}$ such that

$$
\left[\begin{array}{ll}
A^{\prime} & 0 \\
D^{\prime} & I
\end{array}\right]=\left[\begin{array}{ll}
A & 0 \\
D & I
\end{array}\right]\left[\begin{array}{cc}
q & q^{\prime} \\
0 & \tilde{q} \times n
\end{array}\right]
$$

which holds if and only if $q^{\prime}=0, \tilde{q}=I_{m}, A^{\prime}=A q$ and $D^{\prime}=D q$ (since $A$ and $A^{\prime}$ are invertible).

$\underline{(\mathrm{d})}$ : There is a surjective morphism of $\mathbb{C}$-group schemes $P \longrightarrow \mathrm{GL}_{n}$,

$$
\left[\begin{array}{cc}
A_{n \times n} & C_{n \times m} \\
0_{m \times n} & E_{m \times m}
\end{array}\right] \longmapsto A .
$$

This induces the required isomorphism. Notice that the element

$$
\left[\begin{array}{cc}
A_{n \times n} & C_{n \times m} \\
D_{m \times n} & E_{m \times m}
\end{array}\right] \quad \bmod \tilde{P}_{s} \in O_{\mathrm{GL}_{N} / P}^{-} \times P / \tilde{P}_{s}
$$

decomposes (uniquely) as

$$
\left[\begin{array}{cc}
I_{n} & 0 \\
D A^{-1} & I_{m}
\end{array}\right]\left(\left[\begin{array}{cc}
A & C \\
0 & E
\end{array}\right] \bmod \tilde{P}_{s}\right)
$$

Hence it is mapped to $A \bmod Q_{s} \in \mathrm{GL}_{n} / Q_{s}$. Now use (c).

Discussion 2.2.2. Let $\tilde{P}=P_{\left\{i_{1}, \ldots, i_{t}\right\}}$ with $1 \leq i_{1}<\cdots<i_{t} \leq N-1$. Then using Proposition 2.2.1(a) and its proof, $O_{\mathrm{GL}_{N} / \tilde{P}}^{-}$can be identified with the affine space of lower-triangular matrices with possible non-zero entries $x_{i j}$ at row $i$ and column $j$ where $(i, j)$ is such that there exists $l \in\left\{i_{1}, \ldots, i_{t}\right\}$ such that $j \leq l<i \leq N$. To see this, note (from the proof of Proposition 2.2.1(a)) that we are interested in those $(i, j)$ such that the root $\epsilon_{i}-\epsilon_{j}$ belongs to $R^{-} \backslash R_{\tilde{P}}^{-}$. Since $R_{\tilde{P}}^{-}=\bigcap_{k=1}^{t} R_{P_{\widehat{i_{k}}}}^{-}$, we see that we are looking for $(i, j)$ such that $\epsilon_{i}-\epsilon_{j} \in R^{-} \backslash R_{P_{\widehat{l}}}^{-}$for some $l \in\left\{i_{1}, \ldots, i_{t}\right\}$. For the maximal parabolic group $P_{\widehat{l}}$, we have, $R^{-} \backslash R_{P_{\widehat{l}}}^{-}=\left\{\epsilon_{i}-\epsilon_{j} \mid 1 \leq j \leq l<\right.$ $i \leq N\}$. Hence $\operatorname{dim} O_{\mathrm{GL}_{N} / \tilde{P}}^{-}=\left|R^{-} \backslash R_{\tilde{P}}^{-}\right|$.

Let $\alpha=\epsilon_{i}-\epsilon_{j} \in R^{-} \backslash R_{\tilde{P}}^{-}$and $l \in\left\{i_{1}, \ldots, i_{t}\right\}$. Then the Plücker co-ordinate $p_{s_{\alpha}}^{(l)}$ on the Grassmannian $\mathrm{GL}_{N} / P_{\widehat{l}}$ lifts to a regular function on $\mathrm{GL}_{N} / \tilde{P}$, which we denote by the same symbol. Its restriction to $O_{G / \tilde{P}}^{-}$is the the $l \times l$-minor with column indices $\{1,2, \ldots, l\}$ and row indices $\{1, \ldots, j-1, j+1, \ldots, l, i\}$. In particular,

$$
x_{i j}=\left.p_{s_{\alpha}}^{(j)}\right|_{O_{G / \tilde{P}}^{-}} \text {for every } \alpha=\epsilon_{i}-\epsilon_{j} \in R^{-} \backslash R_{\tilde{P}}^{-} .
$$

In general $p_{s_{\alpha}}^{(l)}$ need not be a linear form, or even homogeneous; see the example discussed after Definition 3.2.

Example 2.2.4. Figure 1 shows the shape of $O_{\mathrm{GL}_{N} / \tilde{P}_{s}}^{-}$for some $1 \leq s \leq n-1$. The rectangular region labelled with a circled $\mathrm{D}$ is $O_{\mathrm{GL}}^{-} / P$. The trapezoidal region labelled with a circled $\mathrm{A}$ is $O_{P / \tilde{P}_{s}}^{-}$. In this case, the $x_{i j}$ appearing in $(2.2 .3)$ are exactly those in the regions labelled A and $\mathrm{B}$.

Remark 2.2.5. $X_{\tilde{P}}(w)$ is an irreducible (and reduced) variety of dimension equal to the length of $w$. (Here we use that $w$ is the representative in $W^{\tilde{P}}$.) It can be seen easily that under the natural projection $\mathrm{GL}_{N} / B_{N} \longrightarrow \mathrm{GL}_{N} / \tilde{P}, X_{B_{N}}(w)$ maps birationally onto $X_{\tilde{P}}(w)$ for every $w \in W^{\tilde{P}}$. It is known that Schubert varieties are normal, Cohen-Macaulay and have rational singularities; see, e.g., [BK05, Section 3.4]. 
FREE RESOLUTIONS OF SOME SCHUBERT SINGULARITIES.

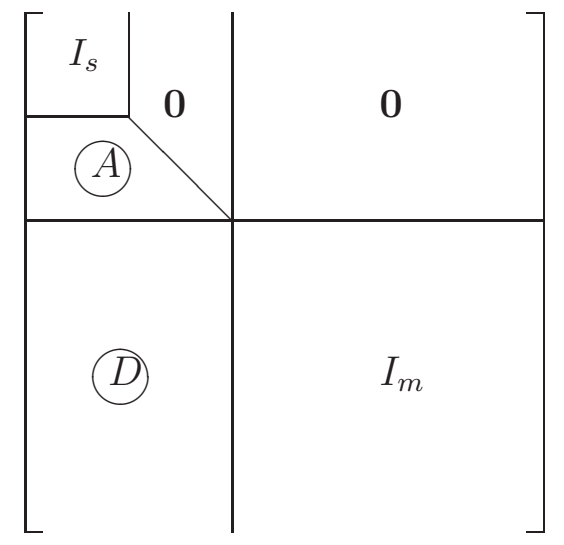

Figure 1. Shape of $O_{\mathrm{GL}_{N} / \tilde{P}_{s}}^{-}$

2.3. Homogeneous bundles and representations. Let $Q$ be a parabolic subgroup of $\mathrm{GL}_{n}$. We collect here some results about homogeneous vector-bundles on $\mathrm{GL}_{n} / Q$. Most of these results are well-known, but for some of them, we could not find a reference, so we give a proof here for the sake of completeness. Online notes of G. Ottaviani [Ott95] and of D. Snow [Sno14] discuss the details of many of these results.

Let $L_{Q}$ and $U_{Q}$ be respectively the Levi subgroup and the unipotent radical of $Q$. Let $E$ be a finite-dimensional vector-space on which $Q$ acts on the right; the vector-spaces that we will encounter have natural right action.

Definition 2.3.1. Define $\mathrm{GL}_{n} \times{ }^{Q} E:=\left(\mathrm{GL}_{n} \times E\right) / \sim$ where $\sim$ is the equivalence relation $(g, e) \sim$ $(g q, e q)$ for every $g \in \mathrm{GL}_{n}, q \in Q$ and $e \in E$. Then $\pi_{E}: \mathrm{GL}_{n} \times{ }^{Q} E \longrightarrow \mathrm{GL}_{n} / Q,(g, e) \mapsto g Q$, is a vector-bundle called the vector-bundle associated to $E$ (and the principal $Q$-bundle $\mathrm{GL}_{n} \longrightarrow$ $\left.\mathrm{GL}_{n} / Q\right)$. For $g \in \mathrm{GL}_{n}, e \in E$, we write $[g, e] \in \mathrm{GL}_{n} \times{ }^{Q} E$ for the equivalence class of $(g, e) \in \mathrm{GL}_{n} \times E$ under $\sim$. We say that a vector-bundle $\pi: \mathbf{E} \longrightarrow \mathrm{GL}_{n} / Q$ is homogeneous if $\mathbf{E}$ has a $\mathrm{GL}_{n}$-action and $\pi$ is $\mathrm{GL}_{n}$-equivariant, i.e, for every $y \in \mathbf{E}, \pi(g \cdot y)=g \cdot \pi(y)$.

In this section, we abbreviate $\mathrm{GL}_{n} \times{ }^{Q} E$ as $\widetilde{E}$. It is known that $\mathbf{E}$ is homogeneous if and only if $\mathbf{E} \simeq \widetilde{E}$ for some $Q$-module $E$. (If this is the case, then $E$ is the fibre of $\mathbf{E}$ over the coset $Q$.) A homogeneous bundle $\widetilde{E}$ is said to be irreducible (respectively indecomposable, completely reducible) if $E$ is a irreducible (respectively indecomposable, completely reducible) $Q$-module. It is known that $E$ is completely reducible if and only if $U_{Q}$ acts trivially and that $E$ is irreducible if and only if additionally it is irreducible as a representation of $L_{Q}$. See [Sno14, Section 5] or [Ott95, Section 10] for the details.

Let $\sigma: \mathrm{GL}_{n} / Q \longrightarrow \widetilde{E}$ be a section of $\pi_{E}$. Let $g \in \mathrm{GL}_{n}$; write $[h, f]=\sigma(g Q)$. There exists a unique $q \in Q$ such that $h=g q$. Let $e=f q^{-1}$. Then $[g, e]=[h, f]$. If $\left[h, f^{\prime}\right]=[h, f]$, then $f^{\prime}=f$, so the assignment $g \mapsto e$ defines a function $\phi: \mathrm{GL}_{n} \longrightarrow E$. This is $Q$-equivariant in the following sense:

$$
\phi(g q)=\phi(g) q, \text { for every } q \in Q \text { and } g \in \mathrm{GL}_{n} .
$$

Conversely, any such map defines a section of $\pi_{E}$. The set of sections $\mathrm{H}^{0}\left(\mathrm{GL}_{n} / Q, \widetilde{E}\right)$ of $\pi_{E}$ is a vector-space with $(\alpha \phi)(g)=\alpha(\phi(g))$ for every $\alpha \in \mathbb{C}, \phi$ a section of $\pi_{E}$ and $g \in \mathrm{GL}_{n}$. It is finite-dimensional. 
Note that $\mathrm{GL}_{n}$ acts on $\mathrm{GL}_{n} / Q$ by multiplication on the left; setting $h \cdot[g, e]=[h g, e]$ for $g, h \in$ $\mathrm{GL}_{n}$ and $e \in E$, we extend this to $\widetilde{E}$. We can also define a natural $\mathrm{GL}_{n}$-action on $\mathrm{H}^{0}\left(\mathrm{GL}_{n} / Q, \widetilde{E}\right)$ as follows. For any map $\phi: \mathrm{GL}_{n} \longrightarrow E$, set $h \circ \phi$ to be the map $g \mapsto \phi\left(h^{-1} g\right)$. If $\phi$ satisfies (2.3.2), then for every $q \in Q$ and $g \in \mathrm{GL}_{n},(h \circ \phi)(g q)=\phi\left(h^{-1} g q\right)=\left(\phi\left(h^{-1} g\right)\right) q=((h \circ \phi)(g)) q$, so $h \circ \phi$ also satisfies (2.3.2). The action of $\mathrm{GL}_{n}$ on the sections is on the left: $\left(h_{2} h_{1}\right) \circ \phi=\left[g \mapsto \phi\left(h_{1}^{-1} h_{2}^{-1} g\right)\right]=$ $\left[g \mapsto\left(h_{1} \circ \phi\right)\left(h_{2}^{-1} g\right)\right]=h_{2} \circ\left(h_{1} \circ \phi\right)$. In fact, $\mathrm{H}^{i}\left(\mathrm{GL}_{n} / Q, \widetilde{E}\right)$ is a $\mathrm{GL}_{n}$-module for every $i$.

Suppose now that $E$ is one-dimensional. Then $Q$ acts on $E$ by a character $\lambda$; we denote the associated line bundle on $\mathrm{GL}_{n} / Q$ by $L_{\lambda}$.

Discussion 2.3.3. Let $Q=P_{i_{1, \ldots, i_{t}}}$, with $1 \leq i_{1}<\cdots<i_{t} \leq n-1$. A weight $\lambda$ is said to be $Q$-dominant if when we write $\lambda=\sum_{i=1}^{n} a_{i} \omega_{i}$ in terms of the fundamental weights $\omega_{i}$, we have, $a_{i} \geq 0$ for all $i \notin\left\{i_{1}, \ldots, i_{t}\right\}$, or equivalently, the associated line bundle (defined above) $L_{\lambda}$ on $Q / B_{n}$ has global sections. If we express $\lambda$ as $\sum_{i=1}^{n} \lambda_{i} \epsilon_{i}$, then $\lambda$ is $Q$-dominant if and only if for every $0 \leq j \leq t, \lambda_{i_{j}+1} \geq \lambda_{i_{j}+2} \geq \cdots \geq \lambda_{i_{j+1}}$ where we set $i_{0}=0$ and $i_{r+1}=n$. We will write $\lambda=\left(\lambda_{1}, \ldots, \lambda_{n}\right)$ to mean that $\lambda=\sum_{i=1}^{n} \lambda_{i} \epsilon_{i}$. Every finite-dimensional irreducible $Q$-module is of the form $\mathrm{H}^{0}\left(Q / B_{n}, L_{\lambda}\right)$ for a $Q$-dominant weight $\lambda$. Hence the irreducible homogeneous vectorbundles on $\mathrm{GL}_{n} / Q$ are in correspondence with $Q$-dominant weights. We describe them now. If $Q=P_{\widehat{n-i}}$, then $\mathrm{GL}_{n} / Q=\mathrm{Gr}_{i, n}$. (Recall that, for us, the $\mathrm{GL}_{n}$-action on $\mathbb{C}^{n}$ is on the right.) On $\mathrm{Gr}_{i, n}$, we have the tautological sequence

$$
0 \longrightarrow \mathcal{R}_{i} \longrightarrow \mathbb{C}^{n} \otimes \mathscr{O}_{\mathrm{Gr}_{i, n}} \longrightarrow \mathcal{Q}_{n-i} \longrightarrow 0
$$

of homogeneous vector-bundles. The bundle $\mathcal{R}_{i}$ is called the tautological sub-bundle (of the trivial bundle $\mathbb{C}^{n}$ ) and $\mathcal{Q}_{n-i}$ is called the tautological quotient bundle. Every irreducible homogeneous bundle on $\mathrm{Gr}_{i, n}$ is of the form $\mathrm{S}_{\left(\lambda_{1}, \cdots, \lambda_{n-i}\right)} \mathcal{Q}_{n-i}^{*} \otimes \mathrm{S}_{\left(\lambda_{n-i+1}, \cdots, \lambda_{n}\right)} \mathcal{R}_{i}^{*}$ for some $P_{\widehat{n-i}}$-dominant weight $\lambda$. Here $\mathrm{S}_{\mu}$ denotes the Schur functor associated to the partition $\mu$. Now suppose that $Q=P_{i_{1}, \ldots, i_{t}}$ with $1 \leq i_{1}<\cdots<i_{t} \leq n-1$. Since the action is on the right, $\mathrm{GL}_{n} / Q$ projects to $\mathrm{Gr}_{n-i, n}$ precisely when $i=i_{j}$ for some $1 \leq j \leq t$. For each $1 \leq j \leq t$, we can take the pull-back of the tautological bundles $\mathcal{R}_{n-i_{j}}$ and $\mathcal{Q}_{i_{j}}$ to $\mathrm{GL}_{n} / Q$ from $\mathrm{GL}_{n} / P_{\widehat{i_{j}}}$. The irreducible homogeneous bundle corresponding to a $Q$-dominant weight $\lambda$ is $\mathrm{S}_{\left(\lambda_{1}, \ldots, \lambda_{i_{1}}\right)} \mathcal{U}_{i_{1}} \otimes \mathrm{S}_{\left(\lambda_{i_{1}+1}, \ldots, \lambda_{i_{2}}\right)}\left(\mathcal{R}_{n-i_{1}} / \mathcal{R}_{n-i_{2}}\right)^{*} \otimes \ldots \otimes$ $\mathrm{S}_{\left(\lambda_{i_{t-1}+1}, \ldots, \lambda_{i_{t}}\right)}\left(\mathcal{R}_{n-i_{t-1}} / \mathcal{R}_{n-i_{t}}\right)^{*} \otimes \mathrm{S}_{\left(\lambda_{i_{t}+1}, \ldots, \lambda_{i_{n}}\right)}\left(\mathcal{R}_{n-i_{t}}\right)^{*}$. See [Wey03, Section 4.1]. Hereafter, we will write $\mathcal{U}_{i}=\mathcal{Q}_{i}^{*}$. Moreover, abusing notation, we will use $\mathcal{R}_{i}, \mathcal{Q}_{i}, \mathcal{U}_{i}$ etc. for these vector-bundles on any (partial) flag varieties on which they would make sense.

A $Q$-dominant weight is called $\left(i_{1}, \ldots, i_{r}\right)$-dominant in [Wey03, p. 114]. Although our definition looks like Weyman's definition, we should keep in mind that our action is on the right. We only have to be careful when we apply the Borel-Weil-Bott theorem (more specifically, Bott's algorithm). In this paper, our computations are done only on Grassmannians. If $\mu$ and $\nu$ are partitions, then $(\mu, \nu)$ will be $Q$-dominant (for a suitable $Q$ ), and will give us the vector-bundle $\mathrm{S}_{\mu} \mathcal{Q}^{*} \otimes \mathrm{S}_{\nu} \mathcal{R}^{*}$ (this is where the right-action of $Q$ becomes relevant) and to compute its cohomology, we will have to apply Bott's algorithm to the $Q$-dominant weight $(\nu, \mu)$. (In [Wey03], one would get $\mathrm{S}_{\mu} \mathcal{R}^{*} \otimes \mathrm{S}_{\nu} \mathcal{Q}^{*}$ and would apply Bott's algorithm to $(\mu, \nu)$.) See, for example, the proof of Proposition 5.4 or the examples that follow it.

Proposition 2.3.5. Let $Q_{1} \subseteq Q_{2}$ be parabolic subgroups and $E$ a $Q_{1}$-module. Let $f: \mathrm{GL}_{n} / Q_{1} \longrightarrow$ $\mathrm{GL}_{n} / Q_{2}$ be the natural map. Then for every $i \geq 0, R^{i} f_{*}\left(\mathrm{GL}_{n} \times{ }^{Q_{1}} E\right)=\mathrm{GL}_{n} \times{ }^{Q_{2}} \mathrm{H}^{i}\left(Q_{2} / Q_{1}, \mathrm{GL}_{n} \times{ }^{Q_{1}}\right.$ $E)$.

Proof. For $Q_{2}$ (respectively, $Q_{1}$ ), the category of homogeneous vector-bundles on $\mathrm{GL}_{n} / Q_{2}$ (respectively, $\left.\mathrm{GL}_{n} / Q_{1}\right)$ is equivalent to the category of finite-dimensional $Q_{2}$-modules (respectively, 
finite-dimensional $Q_{1}$-modules). Now, the functor $f^{*}$ from the category of homogeneous vectorbundles over $\mathrm{GL}_{n} / Q_{2}$ to that over $\mathrm{GL}_{n} / Q_{1}$ is equivalent to the restriction functor $\operatorname{Res}_{Q_{1}}^{Q_{2}}$. Hence their corresponding right-adjoint functors $f_{*}$ and the induction functor $\operatorname{Ind}_{Q_{1}}^{Q_{2}}$ are equivalent; one may refer to [Har77, II.5, p. 110] and [Jan03, I.3.4, 'Frobenius Reciprocity'] to see that these are indeed adjoint pairs. Hence, for homogeneous bundles on $\mathrm{GL}_{n} / Q_{1}, R^{i} f_{*}$ can be computed using $R^{i} \operatorname{Ind}_{Q_{1}}^{Q_{2}}$. On the other hand, note that $\operatorname{Ind}_{Q_{1}}^{Q_{2}}(-)$ is the functor $\mathrm{H}^{0}\left(Q_{2} / Q_{1}, \mathrm{GL}_{n} \times{ }^{Q_{1}}-\right)$ on $Q_{1}$-modules (which follows from [Jan03, I.3.3, Equation (2)]). The proposition now follows.

\section{Properties of Schubert desingularization}

This section is devoted to proving some results on smooth Schubert varieties in partial flag varieties. In Theorem 3.4, we show that opposite cells of certain smooth Schubert varieties in $\mathrm{GL}_{N} / \tilde{P}$ are linear subvarieties of the affine variety $O_{\mathrm{GL}_{N} / \tilde{P}}^{-}$, where $\tilde{P}=\tilde{P}_{s}$ for some $1 \leq s \leq n-1$. Using this, we show in Theorem 3.7 that if $X_{P}(w) \in \mathrm{GL}_{N} / P$ is such that there exists a parabolic subgroup $\tilde{P} \subsetneq P$ such that the birational model $X_{\tilde{P}}(\tilde{w}) \subseteq \mathrm{GL}_{N} / \tilde{P}$ of $X_{P}(w)$ is smooth (we say that $X_{P}(w)$ has a Schubert desingularization if this happens) then the inverse image of $Y_{P}(w)$ inside $X_{\tilde{P}}(\tilde{w})$ is a vector-bundle over a Schubert variety in $P / \tilde{P}$. This will give us a realization of Diagram (1.2).

Recall the following result about the tangent space of a Schubert variety; see [BL00, Chapter 4] for details.

Proposition 3.1. Let $\tau \in W^{\tilde{P}}$. Then the dimension of the tangent space of $X_{\tilde{P}}(\tau)$ at $e_{\mathrm{id}}$ is

$$
\#\left\{s_{\alpha} \mid \alpha \in R^{-} \backslash R_{\tilde{P}}^{-} \text {and } \tau \geq s_{\alpha} \text { in } W / W_{\tilde{P}}\right\} .
$$

In particular, $X_{\tilde{P}}(\tau)$ is smooth if and only if $\operatorname{dim} X_{\tilde{P}}(\tau)=\#\left\{s_{\alpha} \mid \alpha \in R^{-} \backslash R_{\tilde{P}}^{-}\right.$and $\tau \geq$ $s_{\alpha}$ in $\left.W / W_{\tilde{P}}\right\}$.

Definition 3.2. Say that a Schubert variety $X_{\tilde{P}}(\tau)$ has the linearity property if $Y_{\tilde{P}}(\tau)$ (which is defined as $\left.X_{\tilde{P}}(\tau) \cap O_{G / \tilde{P}}^{-}\right)$is a coordinate subspace of $O_{G / \tilde{P}}^{-}$, defined by the vanishing of some of the variables $x_{i j}$ from Discussion 2.2.2.

It is immediate that if $X_{\tilde{P}}(\tau)$ has the linearity property then it is smooth. The converse is not true, as the following example shows. Let $\tau=(2,4,1,3)$ and consider $X_{B}(\tau) \subseteq \mathrm{GL}_{4} / B$. Note that $X_{B}(\tau)$ is smooth. The reflections $(i, j)$ (with $i>j$ ) that satisfy $(i, j) \not \leq \tau$ (in $W=S_{4}$ ) are precisely $(3,1),(4,1)$ and $(4,2)$. For these reflections, we note that the relevant restrictions of the Plücker coordinates to $O_{\mathrm{GL}_{4} / B}^{-}$that vanish on $Y_{\tilde{P}}(\tau)$ are as follows: $\left.p_{(3,1)}^{(1)}\right|_{O_{\mathrm{GL}_{4} / B}^{-}}=x_{31},\left.p_{(4,1)}^{(1)}\right|_{O_{\mathrm{GL}_{4} / B}^{-}}=x_{41}$ and $\left.p_{(4,2)}^{(3)}\right|_{O_{\mathrm{GL}_{4} / B}^{-}}=x_{32} x_{43}-x_{42}$. Hence $Y_{\tilde{P}}(\tau)$ is defined by $x_{31}=0, x_{41}=0, x_{32} x_{43}-x_{42}=0$ as a sub variety of $O_{\mathrm{GL}_{4} / B}^{-}$, showing that $Y_{\tilde{P}}(\tau)$ is a smooth subvariety of $O_{\mathrm{GL}_{4} / B}^{-}$but not a coordinate subspace.

We are interested in the parabolic subgroups $\tilde{P}=\tilde{P}_{s}$ for some $1 \leq s \leq n-1$. Take such a $\tilde{P}$. We will show below that certain smooth Schubert varieties in $\mathrm{GL}_{N} / \widetilde{\tilde{P}}$ have the linearity property. From Discussion 2.2.2 it follows that $\left\{x_{i j} \mid j \leq n\right.$ and $\left.i \geq \max \{j+1, s+1\}\right\}$ is a system of affine coordinates for $O_{\mathrm{GL}_{N} / \tilde{P}}^{-}$.

Notation 3.3. For the remainder of this section we adopt the following notation: Let $w=$ $\left(a_{1}, a_{2}, \ldots, a_{n}\right) \in W^{P}$. Let $r=r(w)$, i.e., the index $r$ such that $a_{r} \leq n<a_{r+1}$. Let $1 \leq s \leq r$. We write $\tilde{P}=\tilde{P}_{s}$. Let $\tilde{w}$ be the minimal representative of $w$ in $W^{\tilde{P}}$. Let $c_{r+1}>\cdots>c_{n}$ be such that 
$\left\{c_{r+1}, \ldots, c_{n}\right\}=\{1, \ldots, n\} \backslash\left\{a_{1}, \ldots, a_{r}\right\} ;$ let $w^{\prime}:=\left(a_{1}, \ldots, a_{r}, c_{r+1}, \ldots, c_{n}\right) \in S_{n}$, the Weyl group of $\mathrm{GL}_{n}$.

Theorem 3.4. With notation as above, suppose that the Schubert variety $X_{\tilde{P}}(\tilde{w})$ of $\mathrm{GL}_{N} / \tilde{P}$ is smooth. Then it has the linearity property.

Proof. Since $a_{1}<\cdots<a_{n}$, we see that for every $j \leq n$ and for every $i \geq \max \left\{a_{j}+1, s+1\right\}$, the reflection $(i, j)$ equals $(1,2, \ldots, j-1, i)$ in $W / W_{P_{\widehat{j}}}$, while $\tilde{w}$ equals $\left(a_{1}, \ldots, a_{j}\right)$. Hence $(i, j)$ is not smaller than $\tilde{w}$ in $W / W_{P_{\widehat{j}}}$, so the Plücker coordinate $p_{(i, j)}^{(j)}$ vanishes on $X_{\tilde{P}}(\tilde{w})$. Therefore for such $(i, j), x_{i j} \equiv 0$ on $Y_{\tilde{P}}(\tilde{w})$, by $(2.2 .3)$.

On the other hand, note that the reflections $(i, j)$ with $j \leq n$ and $i \geq \max \left\{a_{j}+1, s+1\right\}$ are precisely the reflections $s_{\alpha}$ with $\alpha \in R^{-} \backslash R_{\tilde{P}}^{-}$and $\tilde{w} \nsupseteq s_{\alpha}$ in $W / W_{\tilde{P}}$. Since $X_{\tilde{P}}(\tilde{w})$ is smooth, this implies (see Proposition 3.1) that the codimension of $Y_{\tilde{P}}(\tilde{w})$ in $O_{\mathrm{GL}_{N} / \tilde{P}}^{-}$equals

$$
\#\left\{(i, j) \mid j \leq n \text { and } i \geq \max \left\{a_{j}+1, s+1\right\}\right\}
$$

so $Y_{\tilde{P}}(\tilde{w})$ is the linear subspace of $O_{\mathrm{GL}_{N} / \tilde{P}}^{-}$defined by the vanishing of $\left\{x_{i j} \mid j \leq n\right.$ and $i \geq$ $\left.\max \left\{a_{j}+1, s+1\right\}\right\}$.

We have the following immediate corollary to the proof of Theorem 3.4. See Figure 1 for a picture.

Corollary 3.5. Suppose that $X_{\tilde{P}}(\tilde{w})$ is smooth, and identify $O_{G / \tilde{P}}^{-}$with $O_{G / P}^{-} \times O_{P / \tilde{P}}^{-}$(as in Figure 1). Then we have an identification of $Y_{\tilde{P}}(\tilde{w})$ with $\mathcal{V}_{w} \times \mathcal{V}_{w}^{\prime}$, where $\mathcal{V}_{w}$ is the linear subspace of $O_{G / P}^{-}$ (note that $O_{G / P}^{-}$is identified with $M_{m, n}$, the space of all $m \times n$ matrices) given by

$$
x_{i j}=0 \text { if }\left\{\begin{array}{l}
1 \leq j \leq r(w) \text { and for every } i, \\
o r, \\
r(w)+1 \leq j \leq n-1 \text { and } a_{j}-n<i \leq m .
\end{array}\right.
$$

and $\mathcal{V}_{w}^{\prime}$ is the linear subspace of $O_{P / \tilde{P}}^{-}$(being identified with $M_{m, n}$, the space of all $m \times n$ matrices)given by

$$
x_{i j}=0 \text { for every } 1 \leq j \leq r(w) \text { and for every } i \geq \max \left\{a_{j}+1, s+1\right\} .
$$

Proof. As seen in the proof of Theorem 3.4, we have that $Y_{\tilde{P}}(\tilde{w})$ is the subspace of the affine space $O_{G / \tilde{P}}^{-}$given by $x_{i j}=0$ for every $j \leq n$ and for every $i \geq \max \left\{a_{j}+1, s+1\right\}$. This fact together with the identification of $O_{G / \tilde{P}}^{-}$with $O_{G / P}^{-} \times O_{P / \tilde{P}}^{-}$, implies that we have an identification of $Y_{\tilde{P}}(\tilde{w})$ (as a sub variety of $O_{G / P}^{-} \times O_{P / \tilde{P}}^{-}$) with $\mathcal{V}_{w} \times \mathcal{V}_{w}^{\prime}$.

Let $Z_{\tilde{P}}(\tilde{w}):=Y_{P}(w) \times_{X_{P}(w)} X_{\tilde{P}}(\tilde{w})=\left(O_{\mathrm{GL}_{N} / P}^{-} \times P / \tilde{P}\right) \cap X_{\tilde{P}}(\tilde{w})$.

Write $p$ for the composite map $Z_{\tilde{P}}(\tilde{w}) \longrightarrow O_{\mathrm{GL}_{N} / P}^{-} \times P / \tilde{P} \longrightarrow P / \tilde{P}$, where the first map is the inclusion (as a closed subvariety) and the second map is projection. Using Proposition 2.2.1(c) and $(d)$ we see that

$$
p\left(\left[\begin{array}{cc}
A_{n \times n} & 0_{n \times m} \\
D_{m \times n} & I_{m}
\end{array}\right](\bmod \tilde{P})\right)=A\left(\bmod Q_{s}\right) .
$$

( $A$ is invertible by Proposition 2.2.1(b).) 
Using the injective map

$$
A \in B_{n} \mapsto\left[\begin{array}{cc}
A & 0_{n \times m} \\
0_{m \times n} & I_{m}
\end{array}\right] \in B_{N},
$$

$B_{n}$ can be thought of as a subgroup of $B_{N}$. With this identification, we have the following Proposition:

Proposition 3.6. $Z_{\tilde{P}}(\tilde{w})$ is $B_{n}$-stable (for the action on the left by multiplication). Further, $p$ is $B_{n}$-equivariant.

Proof. Let

$$
z:=\left[\begin{array}{cc}
A_{n \times n} & 0_{n \times m} \\
D_{m \times n} & I_{m}
\end{array}\right] \in \mathrm{GL}_{N}
$$

be such that $z \tilde{P} \in Z_{\tilde{P}}(\tilde{w})$. Since $X_{B_{N}}(\tilde{w}) \longrightarrow X_{\tilde{P}}(\tilde{w})$ is surjective, we may assume that $z\left(\bmod B_{N}\right) \in$ $X_{B_{N}}(\tilde{w})$, i.e., $z \in \overline{B_{N} \tilde{w} B_{N}}$. Then for every $A^{\prime} \in B_{n}$

$$
\left[\begin{array}{cc}
A^{\prime} & 0_{n \times m} \\
0_{m \times n} & I_{m}
\end{array}\right] z=\left[\begin{array}{cc}
A^{\prime} A & 0 \\
D & I_{m}
\end{array}\right]=: z^{\prime} .
$$

Then $z^{\prime} \in \overline{B_{N} \tilde{w} B_{N}}$, so $z^{\prime}(\bmod \tilde{P}) \in X_{\tilde{P}}(\tilde{w})$. By Proposition 2.2.1(b), we have that $A$ is invertible, and hence $A A^{\prime}$ is invertible; this implies (again by Proposition 2.2.1(b)) that $z^{\prime}(\bmod \tilde{P}) \in Z_{\tilde{P}}(\tilde{w})$. Thus $Z_{\tilde{P}}(\tilde{w})$ is $B_{n^{-}}$stable. Also, $p\left(A^{\prime} z\right)=p\left(z^{\prime}\right)=A^{\prime} A=A^{\prime} p(z)$. Hence $p$ is $B_{n}$-equivariant.

Theorem 3.7. With notation as above,

(a) The natural map $X_{\tilde{P}}(\tilde{w}) \longrightarrow X_{P}(w)$ is proper and birational. In particular, the map $Z_{\tilde{P}}(\tilde{w}) \longrightarrow Y_{P}(w)$ is proper and birational.

(b) $X_{Q_{s}}\left(w^{\prime}\right)$ is the fibre of the natural map $Z_{\tilde{P}}(\tilde{w}) \longrightarrow Y_{P}(w)$ at $e_{i d} \in Y_{P}(w)$ (w' being as in Notation 3.3).

(c) Suppose that $X_{\tilde{P}}(\tilde{w})$ is smooth. Then $X_{Q_{s}}\left(w^{\prime}\right)$ is the image of $p$. Further, $p$ is a fibration with fibre isomorphic to $\mathcal{V}_{w}$.

(d) Let $X_{\tilde{P}}(\tilde{w})$ be smooth. Then $p$ identifies $Z_{\tilde{P}}(\tilde{w})$ as a sub-bundle of the trivial bundle $O_{\mathrm{GL}_{N} / P}^{-} \times X_{Q_{s}}\left(w^{\prime}\right)$, which arises as the restriction of the vector-bundle on $\mathrm{GL}_{n} / Q_{s}$ associated to the $Q_{s}$-module $\mathcal{V}_{w}$ (which, in turn, is a $Q_{s}$-submodule of $O_{\mathrm{GL}_{N} / P}^{-}$).

We believe that all the assertions above hold without the hypothesis that $X_{\tilde{P}}(\tilde{w})$ is smooth.

Proof. (a): The map $X_{\tilde{P}}(\tilde{w}) \hookrightarrow \mathrm{GL}_{N} / \tilde{P} \longrightarrow \mathrm{GL}_{N} / P$ is proper and its (scheme-theoretic) image is $X_{P}(w)$; hence $X_{\tilde{P}}(\tilde{w}) \longrightarrow X_{P}(w)$ is proper. Birationality follows from the fact that $\tilde{w}$ is the minimal representative of the coset $w \tilde{P}$ (see Remark 2.2.5).

(b): The fibre at $e_{\text {id }} \in Y_{P}(w)$ of the map $Y_{\tilde{P}}(\tilde{w}) \longrightarrow Y_{P}(w)$ is $\{0\} \times \mathcal{V}_{w}^{\prime}\left(\subseteq \mathcal{V}_{w} \times \mathcal{V}_{w}^{\prime}=Y_{\tilde{P}}(\tilde{w})\right)$. Since $Z_{\tilde{P}}(\tilde{w})$ is the closure of $Y_{\tilde{P}}(\tilde{w})$ inside $O_{\mathrm{GL}_{N} / P}^{-} \times P / \tilde{P}$ and $X_{Q_{s}}\left(w^{\prime}\right)$ is the closure of $\mathcal{V}_{w}^{\prime}$ inside $P / \tilde{P}$ (note that as a sub variety of $O_{P / \tilde{P}}^{-}, Y_{Q_{s}}\left(w^{\prime}\right)$ is identified with $\mathcal{V}_{w}^{\prime}$ ), we see that fibre of $Z_{\tilde{P}}(\tilde{w}) \longrightarrow Y_{P}(w)$ at $e_{i d} \in Y_{P}(w)$ is $X_{Q_{s}}\left(w^{\prime}\right)$.

(c) From Theorem 3.4 it follows that

$$
Y_{\tilde{P}}(\tilde{w})=\left\{\left[\begin{array}{cc}
A_{n \times n} & 0_{n \times m} \\
D_{m \times n} & I_{m}
\end{array}\right] \quad \bmod \tilde{P} \mid A \in \mathcal{V}_{w}^{\prime} \text { and } D \in \mathcal{V}_{w}\right\} .
$$

Hence $p\left(Y_{\tilde{P}}(\tilde{w})\right)=\mathcal{V}_{w}^{\prime} \subseteq X_{Q_{s}}\left(w^{\prime}\right)$. Since $Y_{\tilde{P}}(\tilde{w})$ is dense inside $Z_{\tilde{P}}(\tilde{w})$ and $X_{Q_{s}}\left(w^{\prime}\right)$ is closed in $\mathrm{GL}_{n} / Q_{s}$, we see that $p\left(Z_{\tilde{P}_{r}}(\tilde{w})\right) \subseteq X_{Q_{s}}\left(w^{\prime}\right)$. The other inclusion $X_{Q_{s}}\left(w^{\prime}\right) \subseteq p\left(Z_{\tilde{P}_{r}}(\tilde{w})\right)$ follows from (b). Hence, $p\left(Z_{\tilde{P}_{r}}(\tilde{w})\right)$ equals $X_{Q_{s}}\left(w^{\prime}\right)$. 
Next, to prove the second assertion in (c), we shall show that for every $A \in \mathrm{GL}_{n}$ with $A$ $\bmod Q_{s} \in X_{Q_{s}}\left(w^{\prime}\right)$

$$
p^{-1}\left(A \bmod Q_{s}\right)=\left\{\left[\begin{array}{cc}
A & 0_{n \times m} \\
D & I_{m}
\end{array}\right] \quad \bmod \tilde{P}: D \in \mathcal{V}_{w}\right\}
$$

Towards proving this, we first observe that $p^{-1}\left(e_{i d}\right)$ equals $\mathcal{V}_{w}$ (in view of Corollary 3.5). Next, we observe that every $B_{n}$-orbit inside $X_{Q_{s}}\left(w^{\prime}\right)$ meets $\mathcal{V}_{w}^{\prime}\left(=Y_{Q_{s}}\left(w^{\prime}\right)\right)$; further, $p$ is $B_{n}$-equivariant (see Proposition 3.6). The assertion (3.8) now follows.

(d): First observe that for the action of right multiplication by $G L_{n}$ on $\mathcal{O}_{G / P}^{-}$(being identified with $M_{m, n}$, the space of $m \times n$ matrices), $\mathcal{V}_{w}$ is stable; we thus get the homogeneous bundle $\mathrm{GL}_{n} \times{ }^{Q_{s}} \mathcal{V}_{w} \rightarrow \mathrm{GL}_{n} / Q_{s}$ (Definition 2.3.1). Now to prove the assertion about $Z_{\tilde{P}_{s}}(\tilde{w})$ ) being a vector-bundle over $X_{Q_{s}}\left(w^{\prime}\right)$, we will show that there is a commutative diagram given as below, with $\psi$ an isomorphism:

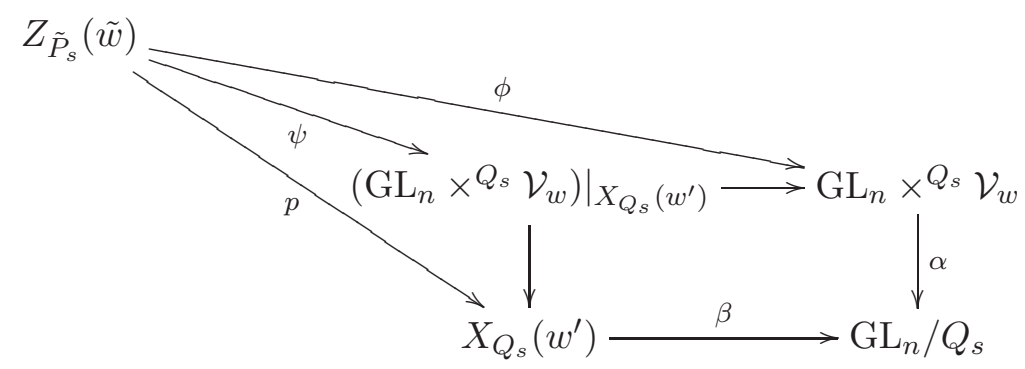

The map $\alpha$ is the homogeneous bundle map and $\beta$ is the inclusion map. Define $\phi$ by

$$
\phi:\left[\begin{array}{cc}
A & 0_{n \times m} \\
D & I_{m}
\end{array}\right] \quad \bmod \tilde{P} \longmapsto(A, D) / \sim .
$$

Using Proposition 2.2.1(c) and (3.8), we conclude the following: $\phi$ is well-defined and injective; $\beta \cdot p=\alpha \cdot \phi$; hence, by the universal property of products, the map $\psi$ exists; and, finally, the injective map $\psi$ is in fact an isomorphism (by dimension considerations).

Corollary 3.9. If $X_{\tilde{P}}(\tilde{w})$ is smooth, then we have the following realization of the diagram in (1.2):

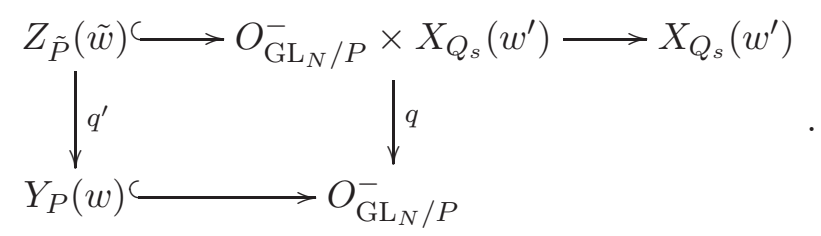

We now describe a class of smooth varieties $X_{\tilde{P}_{s}}(\tilde{w})$ inside $\mathrm{GL}_{N} / \tilde{P}_{s}$.

Proposition 3.10. $X_{\tilde{P}_{s}}(\tilde{w})$ is smooth in the following situations:

(a) $w \in W^{P}$ arbitrary and $s=1$ [Kem71].

(b) $w=\left(n-r+1, \ldots, n, a_{r+1}, \cdots, a_{n-1}, N\right) \in W^{P}$ for some $1 \leq r \leq n-1$ and $s=r$.

Proof. For both (a) and (b): Let $w_{\max } \in W\left(=S_{N}\right)$ be the maximal representative of $\tilde{w}$. We claim that

$$
w_{\max }=\left(a_{s}, a_{s-1}, \ldots, a_{1}, a_{s+1}, a_{s+2}, \ldots, a_{n}, b_{n+1}, \ldots, b_{N}\right) \in W .
$$

Assume the claim. Then $w_{\max }$ is a 4231 - and 3412 -avoiding element of $W$; hence $X_{B_{N}}\left(w_{\max }\right)$ is smooth (see [LS90], [BL00, 8.1.1]). Since $w_{\max }$ is the maximal representative (in $W$ ) of $\tilde{w} \tilde{P}_{s}$, we 
see that $X_{B_{N}}\left(w_{\max }\right)$ is a fibration over $X_{\tilde{P}_{s}}(\tilde{w})$ with smooth fibres $\tilde{P}_{s} / B_{N}$; therefore $X_{\tilde{P}_{s}}(\tilde{w})$ is smooth.

To prove the claim, we need to show that $X_{P_{\hat{i}}}\left(w_{\max }\right)=X_{P_{\hat{i}}}(\tilde{w})$ for every $s \leq i \leq n$ and that $w_{\max }$ is the maximal element of $W$ with this property. This follows, since for every $\tau:=\left(c_{1}, \ldots, c_{N}\right) \in W$ and for every $1 \leq i \leq N, X_{P_{\hat{i}}}(\tau)=X_{P_{\widehat{i}}}\left(\tau^{\prime}\right)$ where $\tau^{\prime} \in W^{P_{\widehat{i}}}$ is the element with $c_{1}, \ldots, c_{i}$ written in the increasing order.

In light of Proposition 3.10(b) we make the following definition. Our concrete descriptions of free resolutions will be for this class of Schubert varieties.

Definition 3.11. Let $1 \leq r \leq n-1$. Let $\mathcal{W}_{r}=\left\{\left(n-r+1, \ldots, n, a_{r+1}, \cdots, a_{n-1}, N\right) \in W^{P}: n<\right.$ $\left.a_{r+1}<\cdots<a_{n-1}<N\right\}$.

The determinantal variety of $(m \times n)$ matrices of rank at most $k$ can be realized as $Y_{P}(w)$, $w=(k+1, \ldots, n, N-k+1, \ldots N) \in \mathcal{W}_{n-k}[$ Ses07, Section 1.6].

Example 3.12. This example shows that even with $r=s, X_{Q_{s}}\left(w^{\prime}\right)$ need not be smooth for arbitrary $w \in W^{P}$. Let $n=m=4$ and $w=(2,4,7,8)$. Then $r=2$; take $s=2$. Then we obtain $w_{\max }=(4,2,7,8,5,6,3,1)$, which has a 4231 pattern.

\section{Free Resolutions}

Kempf-Lascoux-Weyman geometric technique. We summarize the geometric technique of computing free resolutions, following [Wey03, Chapter 5]. Consider Diagram (1.1). There is a natural map $f: V \longrightarrow \operatorname{Gr}_{r, d}$ (where $r=\operatorname{rk}_{V} Z$ and $d=\operatorname{dim} \mathbb{A}$ ) such that the inclusion $Z \subseteq \mathbb{A} \times V$ is the pull-back of the tautological sequence (2.3.4); here $\operatorname{rk}_{V} Z$ denotes the rank of $Z$ as a vectorbundle over $V$, i.e., $\operatorname{rk}_{V} Z=\operatorname{dim} Z-\operatorname{dim} V$. Let $\xi=\left(f^{*} \mathcal{Q}\right)^{*}$. Write $R$ for the polynomial $\operatorname{ring} \mathbb{C}[\mathbb{A}]$ and $\mathfrak{m}$ for its homogeneous maximal ideal. (The grading on $R$ arises as follows. In Diagram (1.1), $\mathbb{A}$ is thought of as the fibre of a trivial vector-bundle, so it has a distinguished point, its origin. Now, being a sub-bundle, $Z$ is defined by linear equations in each fibre; i.e., for each $v \in V$, there exist $s:=\left(\operatorname{dim} \mathbb{A}-\mathrm{rk}_{V} Z\right)$ linearly independent linear polynomials $\ell_{v, 1}, \ldots, \ell_{v, s}$ that vanish along $Z$ and define it. Now $Y=\left\{y \in \mathbb{A}\right.$ : there exists $v \in V$ such that $\left.\ell_{v, 1}(y)=\cdots=\ell_{v, s}(y)=0\right\}$. Hence $Y$ is defined by homogeneous polynomials. This explains why the resolution obtained below is graded.) Let $\mathfrak{m}$ be the homogeneous maximal ideal, i.e., the ideal defining the origin in $\mathbb{A}$. Then:

Theorem 4.1 ([Wey03, Basic Theorem 5.1.2]). With notation as above, there is a finite complex $\left(F_{\bullet}, \partial_{\bullet}\right)$ of finitely generated graded free $R$-modules that is quasi-isomorphic to $\mathbf{R} q_{*}^{\prime} \mathscr{O}_{Z}$, with

$$
F_{i}=\oplus_{j \geq 0} \mathrm{H}^{j}\left(V, \bigwedge^{i+j} \xi\right) \otimes_{\mathbb{C}} R(-i-j),
$$

and $\partial_{i}\left(F_{i}\right) \subseteq \mathfrak{m} F_{i-1}$. Furthermore, the following are equivalent:

(a) $Y$ has rational singularities, i.e. $\mathbf{R} q_{*}^{\prime} \mathscr{O}_{Z}$ is quasi-isomorphic to $\mathscr{O}_{Y}$;

(b) $F_{\bullet}$ is a minimal $R$-free resolution of $\mathbb{C}[Y]$, i.e., $F_{0} \simeq R$ and $F_{-i}=0$ for every $i>0$.

We give a sketch of the proof because one direction of the equivalence is only implicit in the proof of [Wey03, 5.1.3].

Sketch of the proof. One constructs a suitable $q_{*}$-acyclic resolution $\mathscr{I} \bullet$ of the Koszul complex that

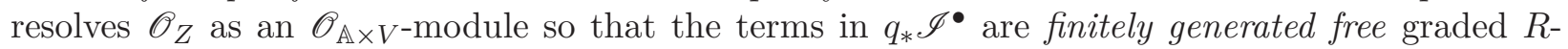
modules. One places the Koszul complex on the negative horizontal axis and thinks of $\mathscr{I} \bullet$ as a second-quadrant double complex, thus to obtain a complex $G_{\bullet}$ of finitely generated free $R$-modules whose homology at the $i$ th position is $R^{-i} q_{*} \mathscr{O}_{Z}$. Then, using standard homological considerations, 
one constructs a subcomplex $\left(F_{\bullet}, \partial_{\bullet}\right)$ of $G_{\bullet}$ that is quasi-isomorphic to $G_{\bullet}$ with $\partial_{i}\left(F_{i}\right) \subseteq \mathfrak{m} F_{i-1}$ (we say that $F_{\bullet}$ is minimal if this happens), and since $\mathrm{H}_{i}\left(G_{\bullet}\right)=0$ for every $|i| \gg 0, F_{i}=0$ for every $|i| \gg 0$. Now using the minimality of $F_{\bullet}$, we see that $R^{i} q_{*} \mathscr{O}_{Z}=0$ for every $i \geq 1$ if and only if $F_{-i}=0$ for every $i \geq 1$. When one of these conditions holds, then $F_{\bullet}$ becomes a minimal free resolution of $q_{*} \mathscr{O}_{Z}$ which is a finitely generated $\mathscr{O}_{Y}$-module, and therefore $q_{*} \mathscr{O}_{Z}=\mathscr{O}_{Y}$ if and only if $q_{*} \mathscr{O}_{Z}$ is generated by one element as an $\mathscr{O}_{Y}$-module if and only if $q_{*} \mathscr{O}_{Z}$ is a generated by one element as an $R$-module if and only if $F_{0}$ is a free $R$-module of rank one if and only if $F_{0}=R(0)$ since $\mathrm{H}^{0}\left(V, \bigwedge^{0} \xi\right) \otimes R$ is a summand of $F_{0}$.

Our situation. We now apply Theorem 4.1 to our situation. We keep the notation of Theorem 3.7. Theorem 4.1 and Corollary 3.9 yield the following result:

Theorem 4.2. Suppose that $X_{\tilde{P}_{s}}(\tilde{w})$ is smooth. Write $\mathcal{U}_{w}$ for the restriction to $X_{Q_{s}}\left(w^{\prime}\right)$ of the vector-bundle on $\mathrm{GL}_{n} / Q_{s}$ associated to the $Q_{s}$-module $\left(O_{\mathrm{GL}_{N} / P}^{-} / \mathcal{V}_{w}\right)^{*}$. (This is the dual of the quotient of $O_{\mathrm{GL}_{N} / P}^{-} \times X_{Q_{s}}\left(w^{\prime}\right)$ by $Z_{\tilde{P}_{s}}(\tilde{w})$.) Then we have a minimal $R$-free resolution $\left(F_{\bullet}, \partial_{\bullet}\right)$ of $\mathbb{C}\left[Y_{P}(w)\right]$ with

$$
F_{i}=\oplus_{j \geq 0} \mathrm{H}^{j}\left(X_{Q_{s}}\left(w^{\prime}\right), \bigwedge^{i+j} \mathcal{U}_{w}\right) \otimes_{\mathbb{C}} R(-i-j) .
$$

In the first case, $Q_{s}=B_{n}$, so $p$ makes $Z_{\tilde{P}_{1}}(\tilde{w})$ a vector-bundle on a smooth Schubert subvariety $X_{B_{1}}\left(w^{\prime}\right)$ of $\mathrm{GL}_{n} / B_{n}$. In the second case, $w^{\prime}$ is the maximal word in $S_{n}$, so $X_{Q_{r}}\left(w^{\prime}\right)=\mathrm{GL}_{n} / Q_{r}$; see Discussion 4.3 for further details.

Computing the cohomology groups required in Theorem 4.2 in the general situation of Kempf's desingularization (Proposition 3.10(a)) is a difficult problem, even though the relevant Schubert variety $X_{B_{n}}\left(w^{\prime}\right)$ is smooth. Hence we are forced to restrict our attention to the subset of $W^{P}$ considered in Proposition 3.10(b).

The stipulation that, for $w \in \mathcal{W}_{r}, w$ sends $n$ to $N$ is not very restrictive. This can be seen in two (related) ways. Suppose that $w$ does not send $n$ to $N$. Then, firstly, $X_{P}(w)$ can be thought of as a Schubert subvariety of a smaller Grassmannian. Or, secondly, $\mathcal{U}_{w}$ will contain the trivial bundle $\mathcal{U}_{n}$ as a summand, so $\mathrm{H}^{0}\left(\mathrm{GL}_{n} / Q_{r}, \xi\right) \neq 0$, i.e., $R(-1)$ is a summand of $F_{1}$. In other words, the defining ideal of $Y_{P}(w)$ contains a linear form.

Discussion 4.3. We give some more details of the situation in Proposition 3.10(b) that will be used in the next section. Let $w=\left(n-r+1, n-r+2, \ldots, n, a_{r+1}, \ldots, a_{n-1}, N\right) \in \mathcal{W}_{r}$. The space of $(m \times n)$ matrices is a $\mathrm{GL}_{n}$-module with a right action; the subspace $\mathcal{V}_{w}$ is $Q_{r}$-stable under this action. Thus $\mathcal{V}_{w}$ is a $Q_{r}$-module, and gives an associated vector-bundle $\left(\mathrm{GL}_{n} \times{ }^{Q_{r}} \mathcal{V}_{w}\right)$ on $\mathrm{GL}_{n} / Q_{r}$. The action on the right of $\mathrm{GL}_{n}$ on the space of $(m \times n)$ matrices breaks by rows; each row is a natural $n$-dimensional representation of $\mathrm{GL}_{n}$. For each $1 \leq j \leq m$, there is a unique $r \leq i_{j} \leq n-1$ such that $a_{i_{j}}<j+n \leq a_{i_{j}+1}$. (Note that $a_{r}=n$ and $a_{n}=N$.) In row $j, \mathcal{V}_{w}$ has rank $n-i_{j}$, and is a sub-bundle of the natural representation. Hence the vector-bundle associated to the $j$ th row of $\mathcal{V}_{w}$ is the pull-back of the tautological sub-bundle (of rank $\left(n-i_{j}\right)$ ) on $\mathrm{Gr}_{n-i_{j}, n}$. We denote this by $\mathcal{R}_{n-i_{j}}$. Therefore $\left(\mathrm{GL}_{n} \times{ }^{Q_{r}} \mathcal{V}_{w}\right)$ is the vector-bundle $\mathcal{R}_{w}:=\bigoplus_{j=1}^{m} \mathcal{R}_{n-i_{j}}$. Let $\mathcal{Q}_{w}:=\bigoplus_{j=1}^{m} \mathcal{Q}_{i_{j}}$ where $\mathcal{Q}_{i_{j}}$ the tautological quotient bundles corresponding to $\mathcal{R}_{n-i_{j}}$. Then the vector-bundle $\mathcal{U}_{w}$ on $\mathrm{GL}_{n} / Q_{r}$ that was defined in Theorem 4.2 is $\mathcal{Q}_{w}^{*}$.

\section{Cohomology of Homogeneous Vector-Bundles}

It is, in general, difficult to compute the cohomology groups $\mathrm{H}^{j}\left(\mathrm{GL}_{n} / Q_{r}, \wedge^{t} \mathcal{U}_{w}\right)$ in Theorem 4.2 for arbitrary $w \in \mathcal{W}_{r}$. In this section, we will discuss some approaches. We believe that this is 
a problem of independent interest. Our method involves replacing $Q_{r}$ inductively by increasingly bigger parabolic subgroups, so we give the general set-up below.

Setup 5.1. Let $1 \leq r \leq n-1$. Let $m_{r}, \ldots, m_{n-1}$ be non-negative integers such that $m_{r}+\cdots+$ $m_{n-1}=m$. Let $Q$ be a parabolic subgroup of $\mathrm{GL}_{n}$ such that $Q \subseteq P_{\hat{i}}$ for every $r \leq i \leq n-1$ such that $m_{i}>0$. We consider the homogeneous vector-bundle $\xi=\oplus_{i=r}^{n-1} \mathcal{U}_{i}^{m_{i}}$ on $\mathrm{GL}_{n} / Q$, We want to compute the vector-spaces $\mathrm{H}^{j}\left(\mathrm{GL}_{n} / Q_{r}, \bigwedge^{t} \xi\right)$.

Lemma 5.2. Let $f: X^{\prime} \longrightarrow X$ be a fibration with fibre some Schubert subvariety $Y$ of some (partial) flag variety. Then $f_{*} \mathscr{O}_{X^{\prime}}=\mathscr{O}_{X}$ and $R^{i} f_{*} \mathscr{O}_{X^{\prime}}=0$ for every $i \geq 1$. In particular, for every locally free coherent sheaves $L$ on $X, \mathrm{H}^{i}\left(X^{\prime}, f^{*} L\right)=\mathrm{H}^{i}(X, L)$ for every $i \geq 0$.

Proof. The first assertion is a consequence of Grauert's theorem [Har77, III.12.9] and the fact (see, for example, [Ses07, Theorem 3.2.1]) that

$$
\mathrm{H}^{i}\left(Y, \mathscr{O}_{Y}\right)= \begin{cases}\mathbb{C}, & \text { if } i=0 \\ 0, & \text { otherwise }\end{cases}
$$

The second assertion follows from the projection formula and the Leray spectral sequence.

Proposition 5.3. Let $m_{i}, r \leq i \leq n-1$ be as in Set-up 5.1. Let $Q^{\prime}=\bigcap_{\substack{r \leq i \leq n-1 \\ m_{i}>0}} P_{\hat{i}}$. Then $\mathrm{H}^{*}\left(\mathrm{GL}_{n} / Q, \bigwedge^{t} \xi\right)=\mathrm{H}^{*}\left(\mathrm{GL}_{n} / Q^{\prime}, \bigwedge^{t} \xi\right)$ for every $t$.

Proof. The assertion follows from Lemma 5.2, noting that $\Lambda^{t} \xi$ on $\mathrm{GL}_{n} / Q$ is the pull-back of $\bigwedge^{t} \xi$ on $\mathrm{GL}_{n} / Q^{\prime}$, under the natural morphism $\mathrm{GL}_{n} / Q \longrightarrow \mathrm{GL}_{n} / Q^{\prime}$.

Proposition 5.4. For all $j, \mathrm{H}^{j}\left(\mathrm{GL}_{n} / Q, \xi\right)=0$.

Proof. We want to show that $\mathrm{H}^{j}\left(\mathrm{GL}_{n} / Q, \mathcal{U}_{i}\right)=0$ for every $r \leq i \leq n-1$ and for every $j$. By Lemma 5.2 (and keeping Discussion 2.3.3 in mind), it suffices to show that $\mathrm{H}^{j}\left(\mathrm{Gr}_{n-i, n}, \mathcal{U}_{i}\right)=0$ for every $r \leq i \leq n-1$ and for every $j$. To this end, we apply the Bott's algorithm [Wey03, (4.1.5)] to the weight

$$
\alpha:=(\underbrace{0, \ldots, 0}_{n-i}, 1, \underbrace{0, \ldots, 0}_{i-1}) .
$$

Note that there is a permutation $\sigma$ such that $\sigma \cdot \alpha=\alpha$. The proposition now follows.

An inductive approach. We are looking for a way to compute $\mathrm{H}^{*}\left(\mathrm{GL}_{n} / Q, \bigwedge^{t} \xi\right)$ for a homogeneous bundle

$$
\xi=\bigoplus_{i \in A} \mathcal{U}_{i}^{\oplus m_{i}}
$$

where $A \subseteq\{r, \ldots, n-1\}$ and $m_{i}>0$ for every $i \in A$. Using Proposition 5.3, we assume that $Q=P_{\widehat{A}}$. (Using Proposition 5.8 below, we may further assume that $m_{i} \geq 2$, but this is not necessary for the inductive argument to work.)

Let $j$ be such that $Q \subseteq P_{\widehat{j}}$ and $\mathcal{Q}_{j}$ (equivalently $\mathcal{U}_{j}$ ) be of least dimension; in other words, $j$ is the smallest element of $A$. If $Q=P_{\widehat{j}}$ (i.e., $|A|=1$ ), then the $\Lambda^{t} \xi$ is completely reducible, and we may use the Borel-Weil-Bott theorem to compute the cohomology groups. Hence suppose that 
$Q \neq P_{\widehat{j}}$; write $Q=Q^{\prime} \cap P_{\widehat{j}}$ non-trivially, with $Q^{\prime}$ being a parabolic subgroup. Consider the diagram

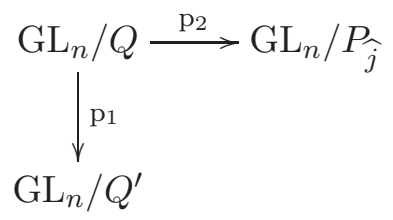

Note that $\bigwedge^{t} \xi$ decomposes as a direct sum of bundles of the form $\left(p_{1}\right)^{*} \eta \otimes\left(p_{2}\right)^{*}\left(\bigwedge^{t_{1}} \mathcal{U}_{j}^{\oplus m_{j}}\right)$ where $\eta$ is a homogeneous bundle on $\mathrm{GL}_{n} / Q^{\prime}$. We must compute $\mathrm{H}^{*}\left(\mathrm{GL}_{n} / Q,\left(p_{1}\right)^{*} \eta \otimes\left(p_{2}\right)^{*}\left(\bigwedge^{t_{1}} \mathcal{U}_{j}^{\oplus m_{j}}\right)\right)$. Using the Leray spectral sequence and the projection formula, we can compute this from $\mathrm{H}^{*}\left(\mathrm{GL}_{n} / Q^{\prime}, \eta \otimes\right.$ $\left.R^{*}\left(p_{1}\right)_{*}\left(p_{2}\right)^{*}\left(\bigwedge^{t_{1}} \mathcal{U}_{j}^{\oplus m_{j}}\right)\right)$. Now $\bigwedge^{t_{1}} \mathcal{U}_{j}^{\oplus m_{j}}$, in turn, decomposes as a direct sum of $\mathrm{S}_{\mu} \mathcal{U}_{j}$, so we must compute $\mathrm{H}^{*}\left(\mathrm{GL}_{n} / Q^{\prime}, \eta \otimes R^{*}\left(p_{1}\right)_{*}\left(p_{2}\right)^{*} \mathrm{~S}_{\mu} \mathcal{U}_{j}\right)$. The Leray spectral sequence and the projection formula respect the various direct-sum decompositions mentioned above. It would follow from Proposition 5.5 below that for each $\mu$, at most one of the $R^{p}\left(p_{1}\right)_{*}\left(p_{2}\right)^{*} \mathrm{~S}_{\mu} \mathcal{U}_{j}$ is non-zero, so the abutment of the spectral sequence is, in fact, an equality.

Proposition 5.5. With notation as above, let $\theta$ be a homogeneous bundle on $\mathrm{GL}_{n} / P_{\widehat{j}}$. Then $R^{i} p_{1 *} p_{2}{ }^{*} \theta$ is the locally free sheaf associated to the vector-bundle $\mathrm{GL}_{n} \times Q^{\prime} \mathrm{H}^{i}\left(Q^{\prime} / Q,\left.p_{2}{ }^{*} \theta\right|_{Q^{\prime} / Q}\right)$ over $\mathrm{GL}_{n} / Q^{\prime}$.

Proof. Follows from Proposition 2.3.5.

We hence want to determine the cohomology of the restriction of $\mathrm{S}_{\mu} \mathcal{U}_{j}$ on $Q^{\prime} / Q$. It follows from the definition of $j$ that $Q^{\prime} / Q$ is a Grassmannian whose tautological quotient bundle and its dual are, respectively, $\left.\mathcal{Q}_{j}\right|_{Q^{\prime} / Q}$ and $\left.\mathcal{U}_{j}\right|_{Q^{\prime} / Q}$. We can therefore compute $\mathrm{H}^{i}\left(Q^{\prime} / Q,\left.\mathrm{~S}_{\mu} \mathcal{U}_{j}\right|_{Q^{\prime} / Q}\right)$ using the Borel-Weil-Bott theorem.

Example 5.6. Suppose that $n=6$ and that $Q=P_{\widehat{\{2,4\}}}$. Then we have the diagram

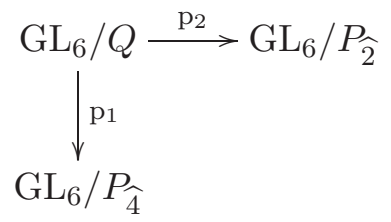

The fibre of $p_{1}$ is isomorphic to $P_{\widehat{4}} / Q$ which is a Grassmannian of two-dimensional subspaces of a four-dimensional vector-space. Let $\mu=\left(\mu_{1}, \mu_{2}\right)$ be a weight. Then we can compute the cohomology groups $\mathrm{H}^{*}\left(P_{\widehat{4}} / Q,\left.\mathrm{~S}_{\mu} \mathcal{U}_{2}\right|_{P_{4} / Q}\right)$ applying the Borel-Weil-Bott theorem [Wey03, (4.1.5)] to the sequence $\left(0,0, \mu_{1}, \mu_{2}\right)$. Note that $\mathrm{H}^{*}\left(P_{\widehat{4}} / Q,\left.\mathrm{~S}_{\mu} \mathcal{U}_{2}\right|_{P_{\widehat{4}}} / Q\right)$ is, if it is non-zero, $\mathrm{S}_{\lambda} W$ where $W$ is a four-dimensional vector-space that is the fibre of the dual of the tautological quotient bundle of $\mathrm{GL}_{4} / P_{\widehat{4}}$ and $\lambda$ is a partition with at most four parts. Hence, by Proposition 5.5, we see that $R^{i}\left(p_{1}\right)_{*}\left(p_{2}\right)^{*} \mathrm{~S}_{\mu} \mathcal{U}_{2}$ is, if it is non-zero, $\mathrm{S}_{\lambda} \mathcal{U}_{4}$ on $\mathrm{GL}_{6} / P_{\widehat{4}}$.

We summarize the above discussion as a theorem:

Theorem 5.7. For $w \in \mathcal{W}_{r}$ the modules in the free resolution of $\mathbb{C}\left[Y_{P}(w)\right]$ given in Theorem 4.2 can be computed.

We end this section with some observations. 
Proposition 5.8. Suppose that there exists $i$ such that $r+1 \leq i \leq n-1$ and such that $\xi$ contains

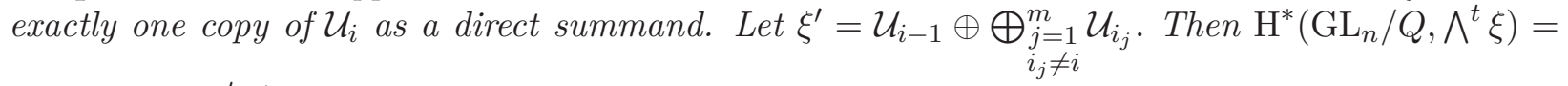
$\mathrm{H}^{*}\left(\mathrm{GL}_{n} / Q, \bigwedge^{t} \xi^{\prime}\right)$ for every $t$.

Proof. Note that $\xi^{\prime}$ is a sub-bundle of $\xi$ with quotient $\mathcal{U}_{i} / \mathcal{U}_{i-1}$. We claim that $\mathcal{U}_{i} / \mathcal{U}_{i-1} \simeq L_{\omega_{i-1}-\omega_{i}}$, where for $1 \leq j \leq n, \omega_{j}$ is the $j$ th fundamental weight. Assume the claim. Then we have an exact sequence

$$
0 \longrightarrow \bigwedge^{t} \xi^{\prime} \longrightarrow \bigwedge^{t} \xi \longrightarrow \bigwedge^{t-1} \xi^{\prime} \otimes L_{\omega_{i-1}-\omega_{i}} \longrightarrow 0
$$

Let $Q^{\prime}=\bigcap_{\substack{r \leq l \leq n-1 \\ l \neq i}} P_{\hat{l}}$; then $Q=Q^{\prime} \cap P_{\widehat{i}}$. Let $p: \mathrm{GL}_{n} / Q \longrightarrow \mathrm{GL}_{n} / Q^{\prime}$ be the natural projection; its fibres are isomorphic to $Q^{\prime} / Q \simeq \mathrm{GL}_{2} / B_{N} \simeq \mathbb{P}^{1}$. Note that $\bigwedge^{t-1} \xi^{\prime} \otimes L_{\omega_{i-1}}$ is the pull-back along $p$ of some vector-bundle on $\mathrm{GL}_{n} / Q^{\prime}$; hence it is constant on the fibres of $p$.

On the other hand, $L_{\omega_{i}}$ is the ample line bundle on $\mathrm{GL}_{n} / P_{\widehat{i}}$ that generates its Picard group, so $L_{-\omega_{i}}$ restricted to any fibre of $p$ is $\mathscr{O}(-1)$. Hence $\Lambda^{t-1} \xi^{\prime} \otimes L_{\omega_{i-1}-\omega_{i}}$ on any fibre of $p$ is a direct sum of copies of $\mathscr{O}(-1)$ and hence it has no cohomology. By Grauert's theorem [Har77, III.12.9], $R^{i} p_{*}\left(\bigwedge^{t-1} \xi^{\prime} \otimes L_{\omega_{i-1}-\omega_{i}}\right)=0$ for every $i$, so, using the Leray spectral sequence, we conclude that $\mathrm{H}^{*}\left(\mathrm{GL}_{n} / Q, \bigwedge^{t-1} \xi^{\prime} \otimes L_{\omega_{i-1}-\omega_{i}}\right)=0$. This gives the proposition.

Now to prove the claim, note that $\mathcal{U}_{i} / \mathcal{U}_{i-1} \simeq\left(\mathcal{R}_{n-i+1} / \mathcal{R}_{n-i}\right)^{*}$. Let $e_{1}, \ldots, e_{n}$ be a basis for $\mathbb{C}^{n}$ such that the subspace spanned by $e_{i}, \ldots, e_{n}$ is $B_{N}$-stable for every $1 \leq i \leq n$. (Recall that we take the right action of $B_{N}$ on $\mathbb{C}^{n}$.) Hence $\mathcal{R}_{n-i+1} / \mathcal{R}_{n-i}$ is the invertible sheaf on which $B_{N}$ acts through the character $\omega_{i}-\omega_{i-1}$, which implies the claim.

Remark 5.9 (Determinantal case). Recall (see the paragraph after Definition 3.11) that $Y_{P}(w)=$ $D_{k}$ if $w=(k+1, \ldots, n, N-k+1, \ldots N) \in \mathcal{W}_{n-k}$. In this case, $\mathcal{U}_{w}=\mathcal{U}_{n-k}^{\oplus(m-k+1)} \oplus \bigoplus_{i=n-k+1}^{n-1} \mathcal{U}_{i}$. Therefore

$$
\mathrm{H}^{*}\left(\mathrm{GL}_{n} / Q_{n-k}, \bigwedge^{*} \xi\right)=\mathrm{H}^{*}\left(\mathrm{GL}_{n} / Q_{n-k}, \bigwedge^{*} \mathcal{U}_{n-k}^{\oplus m}\right)=\mathrm{H}^{*}\left(\mathrm{GL}_{n} / P_{\widehat{n-k}}, \bigwedge^{*} \mathcal{U}_{n-k}^{\oplus m}\right)
$$

where the first equality comes from a repeated application of Proposition 5.8 and the second one follows by Lemma 5.2, applied to the natural map $f: \mathrm{GL}_{n} / Q \longrightarrow \mathrm{GL}_{n} / P_{\widehat{n-k}}$. Hence our approach recovers Lascoux's resolution of the determinantal ideal [Las78]; see also [Wey03, Chapter 6].

\section{EXAMPLES}

We illustrate our approach with two examples. Firstly, we compute the resolution of a determinantal variety using the inductive method from the last section.

Example 6.1 $(n \times m$ matrices of rank $\leq k)$. If $k=1$, then $w=(2, \ldots, n, n+m)$, and, hence, $\xi=\mathcal{U}_{n-1}^{\oplus m}$. Since this would not illustrate the inductive argument, let us take $k=2$.

Consider the ideal generated by the $3 \times 3$ minors of a $4 \times 3$ matrix of indeterminates. It is generated by four cubics, which have a linear relation. Hence minimal free resolution of the quotient ring looks like

$$
0 \longrightarrow R(-4)^{\oplus 3} \longrightarrow R(-3)^{\oplus 4} \longrightarrow R \longrightarrow 0 .
$$


Note that $w=(3,4,6,7)$ and $\xi=\mathcal{U}_{2}^{\oplus 2} \oplus \mathcal{U}_{3}$. Write $G=\mathrm{GL}_{4}$ and $Q=P_{\widehat{2,3}}$. Then $j=2, Q^{\prime}=P_{\widehat{3}}$ and $Q^{\prime} / Q \simeq \mathrm{GL}_{3} / P_{\widehat{2}} \simeq \mathbb{P}^{2}$. Now there is a decomposition

$$
\bigwedge^{t} \xi=\bigoplus_{|\mu| \leq t} \mathrm{~S}_{\mu^{\prime}} \mathbb{C}^{2} \otimes \mathrm{S}_{\mu} \mathcal{U}_{2} \otimes \bigwedge^{t-|\mu|} \mathcal{U}_{3}
$$

Hence we need to consider only $\mu=\left(\mu_{1}, \mu_{2}\right) \leq(2,2)$. On $Q^{\prime} / Q \simeq \mathrm{GL}_{3} / P_{\widehat{2}}$, we would apply the Borel-Weil-Bott theorem [Wey03, (4.1.5)] to the weight $\left(0, \mu_{1}, \mu_{2}\right)$ to compute the cohomology of $\mathrm{S}_{\mu} \mathcal{U}_{j}$. Thus we see that we need to consider only $\mu=(0,0), \mu=(2,0)$ and $\mu=(2,1)$. From this, we conclude that

$$
R^{i}\left(p_{1}\right)_{*}\left(p_{2}\right)^{*}\left(\mathrm{~S}_{\mu^{\prime}} \mathbb{C}^{2} \otimes \mathrm{S}_{\mu} \mathcal{U}_{2}\right)= \begin{cases}\mathscr{O}_{G / P_{\widehat{3}},}, & \text { if } i=0 \text { and } \mu=(0,0) \\ \bigwedge^{2} \mathcal{U}_{3}, & \text { if } i=1 \text { and } \mu=(2,0) ; \\ \left(\bigwedge^{3} \mathcal{U}_{3}\right)^{\oplus 2}, & \text { if } i=1 \text { and } \mu=(2,1) ; \\ 0, & \text { otherwise. }\end{cases}
$$

We have to compute the cohomology groups of $\left(R^{i}\left(p_{1}\right)_{*}\left(p_{2}\right)^{*}\left(\mathrm{~S}_{\mu^{\prime}} \mathbb{C}^{2} \otimes \mathrm{S}_{\mu} \mathcal{U}_{2}\right)\right) \otimes \bigwedge^{t-|\mu|} \mathcal{U}_{3}$ on $G / P_{\widehat{3}}$. Now, $\mathrm{H}^{*}\left(G / P_{\widehat{3}}, \bigwedge^{i} \mathcal{U}_{3}\right)=0$ for every $i>0$. Further

$$
\begin{array}{ll}
\bigwedge^{2} \mathcal{U}_{3} \otimes \mathcal{U}_{3} \simeq \bigwedge^{3} \mathcal{U}_{3} \oplus \mathrm{S}_{2,1} \mathcal{U}_{3} & \text { for } \mu=(2,0) \text { and } t=3 \\
\bigwedge^{2} \mathcal{U}_{3} \otimes \bigwedge^{2} \mathcal{U}_{3} \simeq S_{2,1,1} \mathcal{U}_{3} \oplus \mathrm{S}_{2,2} \mathcal{U}_{3} & \text { for } \mu=(2,0) \text { and } t=4 \\
\bigwedge^{2} \mathcal{U}_{3} \otimes \bigwedge^{3} \mathcal{U}_{3} \simeq \mathrm{S}_{2,2,1} \mathcal{U}_{3} & \text { for }(\mu=(2,0) \text { or } \mu=(2,1)) \text { and } t=5 \\
\bigwedge^{3} \mathcal{U}_{3} \otimes \mathcal{U}_{3} \simeq S_{2,1,1} \mathcal{U}_{3} & \text { for } \mu=(2,1) \text { and } t=4 \\
\bigwedge^{3} \mathcal{U}_{3} \otimes \bigwedge^{3} \mathcal{U}_{3} \simeq S_{2,2,2} \mathcal{U}_{3} & \text { for } \mu=(2,1) \text { and } t=6
\end{array}
$$

Again, by applying the Borel-Weil-Bott theorem [Wey03, (4.1.5)] for $G / P_{\widehat{3}}$, we see that $\mathrm{S}_{2,2} \mathcal{U}_{3}$, $\mathrm{S}_{2,2,1} \mathcal{U}_{3}$ and $\mathrm{S}_{2,2,2} \mathcal{U}_{3}$ have no cohomology. Therefore we conclude that

$$
\mathrm{H}^{j}\left(G / Q, \bigwedge^{t} \xi\right)= \begin{cases}\bigwedge^{0} \mathbb{C}^{\oplus 4}, & \text { if } t=0 \text { and } j=0 \\ \bigwedge^{3} \mathbb{C}^{\oplus 4}, & \text { if } t=3 \text { and } j=2 \\ \left(\bigwedge^{4} \mathbb{C}^{\oplus 4}\right)^{\oplus 3}, & \text { if } t=4 \text { and } j=2 \\ 0, & \text { otherwise. }\end{cases}
$$

These ranks agree with the expected ranks from (6.2).

Example 6.3. Let $n=6, m=6, k=4$ and $w=(5,6,8,9,11,12)$. For this, $Q=P_{\{\widehat{2, \cdots, 5}\}}$ and $\mathcal{U}_{w}=\mathcal{U}_{2}^{\oplus 2} \oplus \mathcal{U}_{3} \oplus \mathcal{U}_{4}^{\oplus 2} \oplus \mathcal{U}_{5}$. After applying Propositions 5.3 and 5.8, we reduce to the situation $Q=P_{\widehat{\{2,4\}}}$ and $\xi=\mathcal{U}_{2}^{\oplus 3} \oplus \mathcal{U}_{4}^{\oplus 3}$. Write $\xi=\left(\mathbb{C}^{3} \otimes_{\mathbb{C}} \mathcal{U}_{2}\right) \oplus\left(\mathbb{C}^{3} \oplus \mathcal{U}_{4}\right)$. Now we project away from 
$\mathrm{GL}_{6} / P_{\widehat{2}}$.

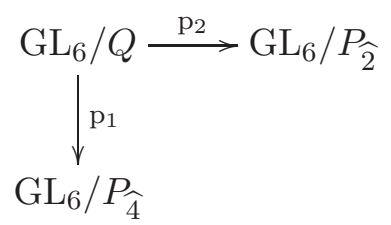

The fibre of $p_{1}$ is isomorphic to $P_{\widehat{4}} / Q$ which is a Grassmannian of two-dimensional subspaces of a four-dimensional vector-space. We use the spectral sequence

$$
\mathrm{H}^{j}\left(G / P_{\widehat{4}}, R^{i} p_{1 *} \bigwedge^{t} \xi\right) \Rightarrow \mathrm{H}^{i+j}\left(G / Q, \bigwedge^{t} \xi\right) .
$$

Observe that $\bigwedge^{t} \xi=\bigoplus_{t_{1}} \bigwedge^{t_{1}}\left(\mathbb{C}^{3} \otimes_{\mathbb{C}} \mathcal{U}_{2}\right) \otimes \bigwedge^{t-t_{1}}\left(\mathbb{C}^{3} \otimes_{\mathbb{C}} \mathcal{U}_{4}\right)$; the above spectral sequence respects this decomposition. Further, using the projection formula, we see that we need to compute

$$
\mathrm{H}^{j}\left(G / P_{\widehat{4}},\left(R^{i} p_{1 *} \bigwedge^{t_{1}}\left(\mathbb{C}^{3} \otimes_{\mathbb{C}} \mathcal{U}_{2}\right)\right) \otimes \bigwedge^{t-t_{1}}\left(\mathbb{C}^{3} \otimes_{\mathbb{C}} \mathcal{U}_{4}\right)\right)
$$

Now, $R^{i} p_{1 *} \bigwedge^{t_{1}}\left(\mathbb{C}^{3} \otimes_{\mathbb{C}} \mathcal{U}_{2}\right)$ is the vector-bundle associated to the $P_{\widehat{4}}$-module $\mathrm{H}^{i}\left(P_{\widehat{4}} / Q, \bigwedge^{t_{1}}\left(\mathbb{C}^{3} \otimes_{\mathbb{C}}\right.\right.$ $\left.\left.\mathcal{U}_{2}\right)\left.\right|_{P_{\widehat{4}} / Q}\right)=\mathrm{H}^{i}\left(P_{\widehat{4}} / Q, \bigwedge^{t_{1}}\left(\left.\mathbb{C}^{3} \otimes \mathbb{C}_{2}\right|_{P_{\widehat{4}} / Q}\right)\right)$. Note that $\left.\mathcal{U}_{2}\right|_{P_{\widehat{4}} / Q}$ is the dual of the tautological quotient bundle of $P_{\widehat{4}} / Q \simeq \mathrm{GL}_{4} / P_{\widehat{2}}$; we denote this also, by abuse of notation, by $\mathcal{U}_{2}$. Note, further, that $\bigwedge^{t_{1}}\left(\mathbb{C}^{3} \otimes \mathbb{C} \mathcal{U}_{2}\right)=\bigoplus_{\mu \vdash t_{1}} \mathrm{~S}_{\mu^{\prime}} \mathbb{C}^{3} \otimes \mathrm{S}_{\mu} \mathcal{U}_{2}$. We need only consider $\mu \leq(3,3)$. From the Borel-Weil-Bott theorem [Wey03, (4.1.5)], it follows that

$$
\mathrm{H}^{i}\left(P_{\widehat{4}} / Q, \mathrm{~S}_{\mu} \mathcal{U}_{2}\right)= \begin{cases}\bigwedge^{0}\left(\mathbb{C}^{\oplus^{4}}\right), & \text { if } i=0 \text { and } \mu=(0,0) ; \\ \bigwedge^{3}\left(\mathbb{C}^{\oplus^{4}}\right), & \text { if } i=2 \text { and } \mu=(3,0) ; \\ \bigwedge^{4}\left(\mathbb{C}^{\oplus^{4}}\right), & \text { if } i=2 \text { and } \mu=(3,1) ; \\ 0, & \text { otherwise. }\end{cases}
$$

Therefore we conclude that

$$
R^{i} p_{1 *} \bigwedge^{t_{1}}\left(\mathbb{C}^{3} \otimes \mathbb{C} \mathcal{U}_{2}\right)= \begin{cases}\mathscr{O}_{\mathrm{GL}_{4} / P_{\widehat{2}},}, & \text { if } i=0 \text { and } t_{1}=0 \\ \bigwedge^{3} \mathcal{U}_{4}, & \text { if } i=2 \text { and } t_{1}=3 \\ \left(\bigwedge^{4} \mathcal{U}_{4}\right)^{\oplus 3}, & \text { if } i=2 \text { and } t_{1}=4 \\ 0, & \text { otherwise. }\end{cases}
$$

Therefore for each pair $\left(t, t_{1}\right)$ at most one column of the summand of the spectral sequence (6.4) is non-zero; hence the abutment in (6.4) is in fact an equality.

Fix a pair $\left(t, t_{1}\right)$ and an integer $l$. Then we have

$$
\begin{aligned}
& \mathrm{H}^{l}\left(G / Q, \bigwedge^{t} \xi\right)=\mathrm{H}^{l}\left(G / P_{\widehat{4}}, \bigwedge^{t}\left(\mathbb{C}^{3} \otimes \mathcal{U}_{4}\right)\right) \oplus \mathrm{H}^{l-2}\left(G / P_{\widehat{4}}, \bigwedge^{3} \mathcal{U}_{4} \otimes \bigwedge^{t-3}\left(\mathbb{C}^{3} \otimes \mathcal{U}_{4}\right)\right) \\
& \oplus \mathrm{H}^{l-2}\left(G / P_{\widehat{4}},\left(\bigwedge^{4} \mathcal{U}_{4}\right)^{\oplus 3} \otimes \bigwedge^{t-4}\left(\mathbb{C}^{3} \otimes \mathcal{U}_{4}\right)\right) .
\end{aligned}
$$

Write $h^{i}(-)=\operatorname{dim}_{\mathbb{C}} \mathrm{H}^{i}(-)$. Note that $\bigwedge^{t}\left(\mathbb{C}^{3} \otimes \mathcal{U}_{4}\right) \simeq \bigoplus_{\lambda \vdash t} \mathrm{~S}_{\lambda^{\prime}} \mathbb{C}^{3} \otimes \mathrm{S}_{\lambda} \mathcal{U}_{4}$, by the Cauchy formula. Write $d_{\mu^{\prime}}=\operatorname{dim}_{\mathbb{C}} \mathrm{S}_{\mu^{\prime}} \mathbb{C}^{\oplus 3}$. Thus, from the above equation, we see, that for every $l$ and for every $t$,

$$
h^{l}\left(\wedge^{t} \xi\right)=\sum_{\mu \vdash t} d_{\mu^{\prime}} h^{l}\left(\mathrm{~S}_{\mu} \mathcal{U}_{4}\right)+\sum_{\mu \vdash t-3} d_{\mu^{\prime}} h^{l-2}\left(\wedge^{3} \mathcal{U}_{4} \otimes \mathrm{S}_{\mu} \mathcal{U}_{4}\right)+3 \sum_{\mu \vdash t-4} d_{\mu^{\prime}} h^{l-2}\left(\wedge^{4} \mathcal{U}_{4} \otimes \mathrm{S}_{\mu} \mathcal{U}_{4}\right)
$$




\begin{tabular}{|c|c|c|c|c|c|c|c|}
\hline$t$ & $h^{0}\left(\wedge^{t} \xi\right)$ & $h^{1}\left(\wedge^{t} \xi\right)$ & $h^{2}\left(\wedge^{t} \xi\right)$ & $h^{3}\left(\wedge^{t} \xi\right)$ & $h^{4}\left(\wedge^{t} \xi\right)$ & $h^{5}\left(\wedge^{t} \xi\right)$ & $h^{6}\left(\wedge^{t} \xi\right)$ \\
\hline 0 & 1 & 0 & 0 & 0 & 0 & 0 & 0 \\
\hline 1 & 0 & 0 & 0 & 0 & 0 & 0 & 0 \\
\hline 2 & 0 & 0 & 0 & 0 & 0 & 0 & 0 \\
\hline 3 & 0 & 0 & 20 & 0 & 0 & 0 & 0 \\
\hline 4 & 0 & 0 & 45 & 0 & 0 & 0 & 0 \\
\hline 5 & 0 & 0 & 36 & 0 & 18 & 0 & 0 \\
\hline 6 & 0 & 0 & 10 & 0 & 53 & 0 & 0 \\
\hline 7 & 0 & 0 & 0 & 0 & 36 & 0 & 0 \\
\hline 8 & 0 & 0 & 0 & 0 & 0 & 0 & 0 \\
\hline 9 & 0 & 0 & 0 & 0 & 0 & 0 & 70 \\
\hline 10 & 0 & 0 & 0 & 0 & 0 & 0 & 153 \\
\hline 11 & 0 & 0 & 0 & 0 & 0 & 0 & 90 \\
\hline 12 & 0 & 0 & 0 & 0 & 0 & 0 & 26 \\
\hline
\end{tabular}

TABLE 1. Ranks of the relevant cohomology groups

(Here the cohomology is calculated over $\mathrm{GL}_{6} / Q$ on the left-hand-side and over $\mathrm{GL}_{6} / P_{\widehat{4}}$ on the right-hand-side.) For any $\mu$, if $d_{\mu^{\prime}} \neq 0$, then $\mu_{1} \leq 3$. Any $\mu$ that contributes a non-zero integer to the right-hand-side of (6.5) has at most four parts and $m_{1} \leq 3$. Further, if $\mathrm{S}_{\lambda} \mathcal{U}_{4}$ is an irreducible summand of a representation on the right-hand-side of (6.5) with non-zero cohomology, then $\lambda$ has at most four parts and is such that $\lambda_{1} \leq 4$. Therefore for $\lambda \leq(4,4,4,4)$, we compute the cohomology using the Borel-Weil-Borel theorem:

$$
\mathrm{H}^{i}\left(G / P_{\widehat{4}}, \mathrm{~S}_{\lambda} \mathcal{U}_{4}\right)= \begin{cases}\wedge^{0}\left(\mathbb{C}^{\oplus 6}\right), & \text { if } i=0 \text { and } \lambda=0 ; \\ \mathrm{S}_{\left(\lambda_{1}-2,1,1, \lambda_{2}, \lambda_{3}, \lambda_{4}\right)}\left(\mathbb{C}^{\oplus 6}\right), & \text { if } i=2, \lambda_{1} \in\{3,4\} \text { and }\left(\lambda_{2}, \lambda_{3}, \lambda_{4}\right) \leq(1,1,1) ; \\ \mathrm{S}_{\left(2,2,2,2, \lambda_{3}, \lambda_{4}\right)}\left(\mathbb{C}^{\oplus 6}\right), & \text { if } i=4, \lambda_{1}=\lambda_{2}=4 \text { and }\left(\lambda_{3}, \lambda_{4}\right) \leq(2,2) ; \\ 0, & \text { otherwise. }\end{cases}
$$

We put these together to compute $h^{l}\left(\wedge^{t} \xi\right)$; the result is listed in Table 1. From this we get the following resolution:

$$
0 \rightarrow R(-12)^{26} \rightarrow R(-11)^{108} \rightarrow \begin{gathered}
R(-6)^{10} \\
\begin{array}{c}
\oplus \\
R(-10)^{153}
\end{array}
\end{gathered} \begin{gathered}
R(-5)^{36} \\
R(-7)^{36} \\
R(-9)^{70}
\end{gathered} \rightarrow \begin{aligned}
& R(-3)^{45} \\
& R(-5)^{53}
\end{aligned} \rightarrow \begin{aligned}
& R(-2)^{20} \\
& R(-4)^{18}
\end{aligned} \rightarrow R \rightarrow 0 .
$$

Note, indeed, that $\operatorname{dim} Y_{Q}(w)=\operatorname{dim} X_{Q}(w)=4+4+5+5+6+6=30$ and that $\operatorname{dim} O_{\mathrm{GL}_{N} / P}^{-}=$ $6 \cdot 6=36$, so the codimension is 6 . Since the variety is Cohen-Macaulay, the length of a minimal free resolution is 6 .

\section{Further REMARKS}

A realization of Lascoux's resolution for determinantal varieties. We already saw in Remark 5.9 that when $Y_{P}(w)=D_{k}$, computing $\mathrm{H}^{*}\left(\mathrm{GL}_{n} / Q_{n-k}, \wedge^{*} \xi\right)$ is reduced, by a repeated application of Proposition 5.8 to computing the cohomology groups of (completely reducible) vector bundles on the Grassmannian $\mathrm{GL}_{n} / P_{\widehat{n-k}}$. We thus realize Lascoux's resolution of the determinantal variety using our approach. 
In this section, we give yet another desingularization of $D_{k}$ (for a suitable choice of the parabolic subgroup) so that the variety $V$ of Diagram (1.2) is in fact a Grassmannian. Recall (the paragraph after Definition 3.11 or Remark 5.9) that $Y_{P}(w)=D_{k}$ if $w=(k+1, \ldots, n, N-k+1, \ldots N) \in \mathcal{W}_{n-k}$. Let $\tilde{P}=P_{\{\hat{n-k, n\}}} \subseteq \mathrm{GL}_{N}$. Let $\tilde{w}$ be the representative of the coset $w \tilde{P}$ in $W^{\tilde{P}}$.

Proposition 7.1. $X_{\tilde{P}}(\tilde{w})$ is smooth and the natural map $X_{\tilde{P}}(\tilde{w}) \longrightarrow X_{P}(w)$ is proper and birational, i.e, $X_{\tilde{P}}(\tilde{w})$ is a desingularization of $X_{P}(w)$.

Proof. The proof is similar to that of Proposition 3.10. Let $w_{\max }=(k+1, \ldots, n, N-k+1, \ldots N, N-$ $k, \ldots, n+1, k, \ldots, 1) \in W$. Then $X_{B_{N}}\left(w_{\max }\right)$ is the inverse image of $X_{\tilde{P}}(\tilde{w})$ under the natural morphism $\mathrm{GL}_{N} / B_{N} \longrightarrow \mathrm{GL}_{N} / \tilde{P}$, and that $w_{\max }$ is a 4231 and 3412-avoiding element of $W=$ $S_{N}$.

We have $P / \tilde{P} \cong \mathrm{GL}_{n} / P_{\widehat{n-k}}$. As in Section 3, we have the following. Denoting by $Z$ the preimage inside $X_{\tilde{P}}(\tilde{w})$ of $Y_{P}(w)$ (under the restriction to $X_{\tilde{P}}(\tilde{w})$ of the natural projection $\left.G / \tilde{P} \rightarrow G / P\right)$, we have $Z \subset O^{-} \times P / \tilde{P}$, and the image of $Z$ under the second projection is $V:=P / \tilde{P}\left(\cong \mathrm{GL}_{n} / P_{\widehat{n-k}}\right)$. The inclusion $Z \hookrightarrow O^{-} \times V$ is a sub-bundle (over $V$ ) of the trivial bundle $O^{-} \times V$. Denoting by $\xi$ the dual of the quotient bundle on $V$ corresponding to $Z$, we have that the homogeneous bundles $\bigwedge^{i+j} \xi$ on $\mathrm{GL}_{n} / P_{\widehat{n-k}}$ are completely reducible, and hence may be computed using Bott's algorithm.

Multiplicity. We describe how the free resolution obtained in Theorem 4.2 can be used to get an expression for the multiplicity multid $(w)$ of the local ring of the Schubert variety $X_{P}(w) \subseteq \mathrm{GL}_{N} / P$ at the point $e_{\mathrm{id}}$. Notice that $Y_{P}(w)$ is an affine neighbourhood of $e_{\mathrm{id}}$. We noticed in Section 4 that $Y_{P}(w)$ is a closed subvariety of $O_{\mathrm{GL}_{N} / P}^{-}$defined by homogeneous equations. In $O_{\mathrm{GL}_{N} / P}^{-}, e_{\mathrm{id}}$ is the origin; hence in $Y_{P}(w)$ it is defined by the unique homogeneous maximal ideal of $\mathbb{C}\left[Y_{P}(w)\right]$. Therefore $\mathbb{C}\left[Y_{P}(w)\right]$ is the associated graded ring of the local ring of $\mathbb{C}\left[Y_{P}(w)\right]$ at $e_{\text {id }}$ (which is also the local ring of $X_{P}(w)$ at $\left.e_{\mathrm{id}}\right)$. Hence mult $\mathrm{id}_{\mathrm{i}}(w)$ is the normalized leading coefficient of the Hilbert series of $\mathbb{C}\left[Y_{P}(w)\right]$.

Observe that the Hilbert series of $\mathbb{C}\left[Y_{P}(w)\right]$ can be obtained as an alternating sum of the Hilbert series of the modules $F_{i}$ in Theorem 4.2. Write $h^{j}(-)=\operatorname{dim}_{\mathbb{C}} \mathrm{H}^{j}\left(X_{Q_{s}}\left(w^{\prime}\right),-\right)$ for coherent sheaves on $X_{Q_{s}}\left(w^{\prime}\right)$. Then Hilbert series of $\mathbb{C}\left[Y_{P}(w)\right]$ is

$$
\frac{\sum_{i=0}^{m n} \sum_{j=0}^{\operatorname{dim}}(-1)^{i} h^{j}\left(\bigwedge^{i+j} \mathcal{U}_{w}\right) t^{i+j}}{(1-t)^{m n}} .
$$

We may harmlessly change the range of summation in the numerator of (7.2) to $-\infty<i, j<\infty$; this is immediate for $j$, while for $i$, we note that the proof of Theorem 4.1 implies that $h^{j}\left(\bigwedge^{i+j} \mathcal{U}_{w}\right)=0$ for every $i<0$ and for every $j$. Hence we may write the numerator of (7.2) as (with $k=i+j$ )

$$
\sum_{k=0}^{\infty}(-1)^{k} t^{k} \sum_{j=0}^{\infty}(-1)^{j} h^{j}\left(\wedge^{k} \mathcal{U}_{w}\right)=\sum_{k=0}^{\mathrm{rk} \mathcal{U}_{w}}(-1)^{k} \chi\left(\wedge^{k} \mathcal{U}_{w}\right) t^{k}
$$

Since $\wedge^{k} \mathcal{U}_{w}$ is also a $T_{n}$-module, where $T_{n}$ is the subgroup of diagonal matrices in $\mathrm{GL}_{n}$, one may decompose $\wedge^{k} \mathcal{U}_{w}$ as a sum of rank-one $T_{n}$-modules and use the Demazure character formula to compute the Euler characteristics above.

It follows from generalities on Hilbert series (see, e.g., [BH93, Section 4.1]) that the polynomial in $(7.3)$ is divisible by $(1-t)^{c}$ where $c$ is the codimension of $Y_{P}(w)$ in $O_{\mathrm{GL}_{N} / P}^{-}$, and that after we 
divide it and substitute $t=1$ in the quotient, we get $\operatorname{mult}_{\mathrm{id}}(w)$. This gives an expression for $e_{\mathrm{id}}(w)$ apart from those of [LW90, KL04].

Castelnuovo-Mumford Regularity. Since $\mathbb{C}\left[Y_{P}(w)\right]$ is a graded quotient ring of $\mathbb{C}\left[O_{\mathrm{GL}_{N} / P}^{-}\right]$, it defines a coherent sheaf over the corresponding projective space $\mathbb{P}^{m n-1}$.

Let $F$ be a coherent sheaf on $\mathbb{P}^{n}$. The Castelnuovo-Mumford regularity of $F$ (with respect to $\left.\mathscr{O}_{\mathbb{P}^{n}}(1)\right)$ is the smallest integer $r$ such that $\mathrm{H}^{i}\left(\mathbb{P}^{n}, F \otimes \mathscr{O}_{\mathbb{P}^{n}}(r-i)\right)=0$ for every $1 \leq i \leq n$; we denote it by reg $F$. Similarly, if $R=\mathbb{k}\left[x_{0}, \ldots, x_{n}\right]$ be a polynomial ring over a field $\mathbb{k}$ with $\operatorname{deg} x_{i}=1$ for every $i$ and $M$ is a finitely generated graded $R$-module, the Castelnuovo-Mumford regularity of $M$ to be the smallest integer $r$ such that $\left(\mathrm{H}_{\left(x_{0}, \ldots, x_{n}\right)}^{i}(M)\right)_{r+1-i}=0$ for every $0 \leq i \leq n+1$; we denote it by reg $M$. (Here $\mathrm{H}_{\left(x_{0}, \ldots, x_{n}\right)}^{i}(M)$ is the $i$ local cohomology module of $M$, and is a graded $R$-module.) It is known that $\operatorname{reg} F=\operatorname{reg}\left(\oplus_{i \in \mathbb{Z}} \mathrm{H}^{0}\left(\mathbb{P}^{n}, F \otimes \mathscr{O}_{\mathbb{P}^{n}}(i)\right)\right)$ for every coherent sheaf $F$ and that if $\operatorname{depth} M \geq 2$, then $\operatorname{reg} M=\operatorname{reg} \widetilde{M}$. See [Eis05, Chapter 4] for details.

Proposition 7.4. In the notation of Diagram (1.1), $\operatorname{reg} \mathbb{C}[Y]=\max \left\{j: \mathrm{H}^{j}\left(V, \wedge^{*} \xi\right) \neq 0\right\}$.

Proof. Let $R=\mathbb{C}[\mathbb{A}]$. It is known that $\operatorname{reg} M=\max \left\{j: \operatorname{Tor}_{i}^{R}(\mathbb{k}, M)_{i+j} \neq 0\right.$ for some $\left.i\right\}$; see $\left[\right.$ Eis05, Chapter 4] for a proof. The proposition now follows from noting that $\operatorname{Tor}_{i}^{R}(\mathbb{C}, \mathbb{C}[Y])_{i+j} \simeq$ $\mathrm{H}^{j}\left(V, \wedge^{i+j} \xi\right)$ (Theorem 4.2).

Now let $w=\left(n-r+1, n-r+2, \ldots, n, a_{r+1}, \ldots, a_{n-1}, N\right) \in \mathcal{W}_{r}$. We would like to determine $\operatorname{reg} \mathbb{C}\left[Y_{P}(w)\right]=\max \left\{j: \mathrm{H}^{j}\left(\mathrm{GL}_{n} / Q_{r}, \wedge^{*} \mathcal{U}_{w}\right) \neq 0\right\}$. Let $a_{r}=n$ and $a_{n}=N$. For $r \leq i \leq n-1$, define $m_{i}=a_{i+1}-a_{i}$. Note that $\mathcal{U}_{i}$ appears in $\mathcal{U}_{w}$ with multiplicity $m_{i}$ and that $m_{i}>0$. Based on the examples that we have calculated, we have the following conjecture.

Conjecture 7.5. With notation as above,

$$
\operatorname{reg} \mathbb{C}\left[Y_{P}(w)\right]=\sum_{i=r}^{n-1}\left(m_{i}-1\right) i .
$$

(Note that since $Y_{P}(w)$ is Cohen-Macaulay, $\operatorname{reg} \mathbb{C}\left[Y_{P}(w)\right]=\operatorname{reg} \mathscr{O}_{Y_{P}(w)}$.) Consider the examples in Section 6. In Example 6.1, $m_{2}=2, m_{3}=1$ and $\operatorname{reg} \mathbb{C}\left[Y_{P}(w)\right]=(2-1) 2+0=2$. In Example 6.3, $m_{2}=m_{4}=2$ and $m_{3}=m_{5}=1$, so $\operatorname{reg} \mathbb{C}\left[Y_{P}(w)\right]=(2-1) 2+0+(2-1) 4+0=6$, which in deed is the case, as we see from Table 1.

\section{REFERENCES}

[BH93] W. Bruns and J. Herzog. Cohen-Macaulay rings, volume 39 of Cambridge Studies in Advanced Mathematics. Cambridge University Press, Cambridge, 1993. 21

[BK05] M. Brion and S. Kumar. Frobenius splitting methods in geometry and representation theory, volume 231 of Progress in Mathematics. Birkhäuser Boston, Inc., Boston, MA, 2005. 6

[BL00] S. Billey and V. Lakshmibai. Singular loci of Schubert varieties, volume 182 of Progress in Mathematics. Birkhäuser Boston Inc., Boston, MA, 2000. 3, 9, 12

[Bor91] A. Borel. Linear algebraic groups, volume 126 of Graduate Texts in Mathematics. Springer-Verlag, New York, second edition, 1991. 3

[Eis05] D. Eisenbud. The Geometry of Syzygies, volume 229 of Graduate Texts in Mathematics. Springer-Verlag, New York, 2005. A second course in commutative algebra and algebraic geometry. 22

[M2] D. R. Grayson and M. E. Stillman. Macaulay 2, a software system for research in algebraic geometry, 2006. Available at http://www.math.uiuc.edu/Macaulay2/. 3

[Har77] R. Hartshorne. Algebraic geometry. Springer-Verlag, New York, 1977. Graduate Texts in Mathematics, No. 52. $9,15,17$ 
[Jan03] J. C. Jantzen. Representations of algebraic groups, volume 107 of Mathematical Surveys and Monographs. American Mathematical Society, Providence, RI, second edition, 2003. 3, 9

[Kem71] G. Kempf. Schubert methods with an application to algebraic curves., 1971. Math. Centrum, Amsterdam, Afd. zuivere Wisk. ZW 6/71, 18 p. (1971). 12

[KL04] V. Kreiman and V. Lakshmibai. Multiplicities of singular points in Schubert varieties of Grassmannians. In Algebra, arithmetic and geometry with applications (West Lafayette, IN, 2000), pages 553-563. Springer, Berlin, 2004. 22

[Las78] A. Lascoux. Syzygies des variétés déterminantales. Adv. in Math., 30(3):202-237, 1978. 1, 17

[LS90] V. Lakshmibai and B. Sandhya. Criterion for smoothness of Schubert varieties in $\operatorname{Sl}(n) / B$. Proc. Indian Acad. Sci. Math. Sci., 100(1):45-52, 1990. 12

[LW90] V. Lakshmibai and J. Weyman. Multiplicities of points on a Schubert variety in a minuscule $G / P$. Adv. Math., 84(2):179-208, 1990. 22

[OR06] G. Ottaviani and E. Rubei. Quivers and the cohomology of homogeneous vector bundles. Duke Math. J., 132(3):459-508, 2006. 3

[Ott95] G. Ottaviani. Rational Homogeneous Varieties, 1995. SMI Course in Algebraic Geometry, Cortona (Italy); available at http://www.math.unifi.it/ ottavian/public.html. 7

[Ses07] C. S. Seshadri. Introduction to the theory of standard monomials, volume 46 of Texts and Readings in Mathematics. Hindustan Book Agency, New Delhi, 2007. With notes by Peter Littelmann and Pradeep Shukla, Appendix A by V. Lakshmibai, Revised reprint of lectures published in the Brandeis Lecture Notes series. 3, 13, 15

[Sno14] D. Snow. Homogeneous Vector Bundles, 2014. Available at http://www3.nd.edu/ snow/Papers/HomogVB.pdf. 7

[LiE] M. A. A. van Leeuwen, A. M. Cohen, and B. Lisser. LiE, a computer algebra package for Lie group computations, 2000. Available at http://wwwmathlabo.univ-poitiers.fr/ maavl/LiE/index.html. 3

[Wey03] J. Weyman. Cohomology of vector bundles and syzygies, volume 149 of Cambridge Tracts in Mathematics. Cambridge University Press, Cambridge, 2003. 1, 8, 13, 15, 16, 17, 18, 19

Chennai Mathematical Institute, Siruseri, Tamilnadu 603103. India

E-mail address: mkummini@cmi.ac.in

Northeastern University, Boston, Massachusetts. USA.

E-mail address: lakshmibai@neu.edu

Chennai Mathematical Institute, Siruseri, Tamilnadu 603103. India

E-mail address: pramath@cmi.ac.in

Chennai Mathematical Institute, Siruseri, Tamilnadu 603103. India

E-mail address: css@cmi.ac.in 